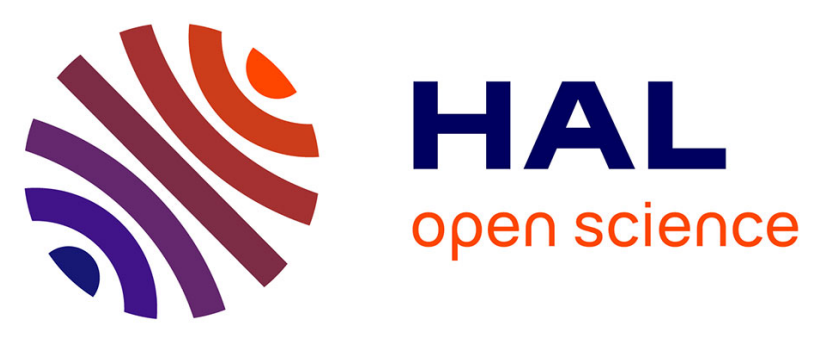

\title{
Design of a fast field-cycling magnetic resonance imaging system, characterization and methods for relaxation dispersion measurements around $1.5 \mathrm{~T}$
}

Nicolas Chanet, Geneviève Guillot, Georges Willoquet, Laurène Jourdain, Rose-Marie Dubuisson, Gaël Reganha, Ludovic de Rochefort

\section{To cite this version:}

Nicolas Chanet, Geneviève Guillot, Georges Willoquet, Laurène Jourdain, Rose-Marie Dubuisson, et al.. Design of a fast field-cycling magnetic resonance imaging system, characterization and methods for relaxation dispersion measurements around 1.5 T. Review of Scientific Instruments, 2020, 91 (2), pp.024102. 10.1063/1.5128851 . hal-03000885

\section{HAL Id: hal-03000885 https://hal.science/hal-03000885}

Submitted on 20 Nov 2020

HAL is a multi-disciplinary open access archive for the deposit and dissemination of scientific research documents, whether they are published or not. The documents may come from teaching and research institutions in France or abroad, or from public or private research centers.
L'archive ouverte pluridisciplinaire HAL, est destinée au dépôt et à la diffusion de documents scientifiques de niveau recherche, publiés ou non, émanant des établissements d'enseignement et de recherche français ou étrangers, des laboratoires publics ou privés. 


\title{
Design of a fast field-cycling magnetic resonance imaging system, characterization and methods for relaxation dispersion measurements around $1.5 \mathrm{~T}$
}

\author{
Authors \\ Nicolas Chanet $^{1 *}$, Geneviève Guillot ${ }^{*}$, Georges Willoquet ${ }^{1}$, Laurène Jourdain ${ }^{1}$, Rose-Marie \\ Dubuisson $^{1}$, Gaël Reganha ${ }^{1}$, Ludovic de Rochefort ${ }^{2 *}$
}

\begin{abstract}
Affiliations
${ }^{1}$ Imagerie par Résonance Magnétique Médicale et Multi-Modalités (UMR8081) IR4M, CNRS, Université Paris-Sud, Université Paris-Saclay, Orsay, France.

${ }^{2}$ Aix-Marseille Univ, CNRS, CRMBM (Center for Magnetic Resonance in Biology and Medicine - UMR 7339), Marseille, France. ludovic.de-rochefort@univ-amu.fr
\end{abstract}

*these authors contributed equally to this work

\section{Contact information}

Ludovic de Rochefort, $\mathrm{PhD}$

Center for Magnetic Resonance in Biology and Medicine - UMR 7339, CNRS - Aix Marseille

Université

13385 Marseille, France

Tel: +33491386262

E-mail: ludovic.de-rochefort@univ-amu.fr

This accepted manuscript is published as:

Chanet N, Guillot G, Willoquet G, Jourdain L, Dubuisson RM, Reganha G, de Rochefort L. Design of a fast fieldcycling magnetic resonance imaging system, characterization and methods for relaxation dispersion measurements around 1.5 T. The Review of scientific instruments 2020;91(2):024102. 


\begin{abstract}
The dependence of nuclear magnetic resonance relaxation rate with the magnetic field has been widely studied, in particular in biomedical situations with the objectives to better understand the underlying microscopic mechanisms in tissues and provide biomarkers of diseases. By combining fast-field cycling (FFC) and magnetic resonance imaging (MRI), it is possible to provide localized relaxation dispersion measurements in heterogeneous systems, with recent demonstrations in solutions, biological samples, human and small animals. We report here the developments and performances of a device designed for small animal FFC-MRI comprising a resistive insert technology operating inside a 1.5 T MRI system. Specific measurement methods were developed to characterize the system efficiency, response time, homogeneity, stability and compensation. By adding a non-linear element in the system and using a dual amplifier strategy, it is shown that large field offsets can be produced during relaxation periods while maintaining precise field control during detection periods. The measurement of longitudinal NMRD dispersion profiles in a range of 1.08 to $1.92 \mathrm{~T}$ are reported, essentially displaying a linear variation in this range for common MRI contrast agents. The slopes of both the longitudinal and transverse relaxation dispersion profiles at $1.5 \mathrm{~T}$ are measured and validated, extending the capabilities of previous approaches. The performances of a longitudinal relaxation dispersion mapping method is finally reported, opening the way to quantitative preclinical dispersion imaging studies at high FFC-MRI field.
\end{abstract}




\section{INTRODUCTION}

Nuclear magnetic resonance (NMR) relaxometry consists in the measurement of longitudinal and transverse nuclear magnetization relaxation times (respectively $T_{1}$ and $T_{2}$ ) or equivalently their inverse, the relaxation rates $R_{1}$ and $R_{2}{ }^{1,2}$. The relaxation rates vary with the magnetic field $B_{0}$ due to their links to microscopic motions characterized by spectral densities ${ }^{3-5}$ that are frequency-dependent. The magnetic field dependence of the relaxation rate is referred to as a NMR dispersion (NMRD) profile, and can be measured advantageously with relaxometers that allow the main magnetic field $\mathrm{B}_{0}$ to vary rapidly as compared to relaxations times. Shifting rapidly $\mathrm{B}_{0}$ during relaxation can be done using fast-field cycling (FFC) or sample-shuttling relaxometers ${ }^{6-11}$. The main principle of the former is to vary the electrical current in resistive magnets, while the latter principle is to move the sample at different stray field positions. Microscopic motions in a variety of molecular systems and media can be studied with relaxometry (such as proteins, polymers, or porous media) such that applications of NMR relaxometry range from chemistry, through rock characterization, to biomedical sciences.

In biomedical situations, proton ${ }^{1} \mathrm{H}$ relaxation is most often studied, with the objective to characterize the interactions of water molecules with their local environment in tissues ${ }^{12}$. Indeed, water relaxation in tissues is field-dependent comprising separable mechanisms encompassing interactions with longlived-protein binding sites and diffusion at macromolecular interfaces ${ }^{13}$. Additional relaxation mechanisms can be observed either with endogenous metalloproteins ${ }^{14}$ or exogenous contrast agents based on paramagnetic and superparamagnetic compounds ${ }^{15,16}$. Interaction with quadrupole nuclei, such as with ${ }^{14} \mathrm{~N}$, can also lead to quadrupole relaxation enhancement ${ }^{16-18}$ most often seen at low frequencies, providing information on the interactions with proteins.

Field-cycling relaxometers have limited ability to localize NMR signal ${ }^{7}$ while it is needed to characterize heterogeneous samples. While magnetic resonance imaging (MRI) systems can be used to localize nuclear magnetization and map relaxation rates, it can usually be done only at a fixed magnetic field $\mathrm{B}_{0}{ }^{19}$. To be able to perform localized relaxation measurements in a varying and resolved range of magnetic fields, combined FFC-MRI systems and associated measurement methods have been developed ${ }^{20-33}$, providing new means to access contrasts based on NMR dispersion, enabling a better understanding of contrast mechanisms, and potentially leading to more specific biomedical markers of diseases. In the last decade ${ }^{33}$, clinical FFC-MRI was shown feasible at low field (up to $0.2 \mathrm{~T}$ ) ${ }^{29,30}$, and pre-clinical FFC-MRI at high field (up to $3 \mathrm{~T}$ ) $20,24,31-34$ with promising initial in vivo images obtained of longitudinal relaxation dispersion reflecting local interactions of water protons in tissues ${ }^{35}$. 
There are several technical challenges (such as rapidity, stability, homogeneity) to obtain MR images of high quality combined with dispersive contrasts accessible by fast field cycling. MRI by itself requires a high magnetic field stability for imaging, but a FFC-MRI system must also be designed to vary rapidly the magnetic field as compared to longitudinal or transverse relaxation times. A solution is to insert a resistive coil performing the fast $\mathrm{B}_{0}$ variations during relaxation periods into a conventional superconducting high-field MRI system benefiting from a larger polarization together with more stable and homogeneous field during detection. However, interactions with the MRI scanner and the insert are usually observed. Indeed, the systems are then inductively coupled, such that current variations in the insert induce eddy-currents in the conductive structures of the host system, that are dissipated as heat in its resistive structures. In the various setups developed involving an inserted coil into a superconductive magnet ${ }^{20,24,28,32}$, shielding coils were used in order to reduce eddy currents and the risk of quench, while at the same time complexifying the coil design and construction, and reducing the coil efficiency. In all cases, eddy-currents, while reduced, were still present, still necessitating correction strategies. This could be successfully achieved with the use of shielding/compensation coils $^{28}$ and of eddy-current correction techniques ${ }^{25,36}$. Instead of compensating the field directly, another strategy for eddy-current correction relies on real-time transmit and receive radiofrequency modulation ${ }^{25}$, locking them with the instantaneous NMR frequency whose temporal evolution can be pre-calibrated and should be reproducible after a given $\mathrm{B}_{0}$ offset pulse. As we will see below, the setup described here does not require a shielding coil and compensates for eddy-currents by directly injecting a counter current waveform within the insert.

Another major technical issue is the stability of the current in the insert coil when RF pulses are applied and NMR signals collected. Indeed, when using systems capable of large field offsets, current fluctuations are producing random NMR frequency and consequently phase fluctuations during excitation and acquisition of the multiple signals collected for image generation which can reduce image quality and localization precision. It is usually considered that field perturbations higher than $10^{-9}$ T.s cause noticeable signal alterations ${ }^{37}$, thus are source of image artefacts and loss of signal-tonoise ratio. MRI acquisition windows are typically in the millisecond range, thus the corresponding magnetic field stability should be better than $1 \mu \mathrm{T}$. If the desired field offset is in the range of $1 \mathrm{~T}$, the relative accuracy for current in the insert coil should thus be lower than $10^{-6}$ to avoid image artefacts. To avoid fluctuations during the acquisition period, which uses the stable main field of the superconducting magnet, a possible strategy is to switch to a highly resistive state, which in the extreme limit corresponds to opening the coil circuit by disabling the amplifier ${ }^{32}$. A drawback is that 
an additional delay is needed to enable again the power supply, preventing the use of high duty cycles. Another approach is to switch the amplifier load between the insert coil and an inductance-matched dummy load using an active element composed of fast high-power solid state switches ${ }^{28}$.

Finally, a good magnetic field homogeneity is always required during the readout period ${ }^{38}$. The airtissue magnetic susceptibility difference provides a typical order of magnitude of the targeted homogeneity range of $\pm 10 \mathrm{ppm}$, as there is no need to design more homogeneous systems. Homogeneity requirements during relaxation periods essentially depends on the expected field resolution (typically less than 1-2\% variations across the field of view ${ }^{24,28,32}$, of the targeted field range). The inhomogeneity scales with the current such that inserts designed to operate inside a high field system and that are not intended to reach low or very low fields consequently needs not to be very homogeneous ${ }^{28}$.

Most FFC studies focussed on the measurement or on the exploitation of the dispersion of longitudinal relaxation. The traditional way to do so is to use several evolution times and several evolution fields in an inversion- or saturation-recovery sequence (see Fig. 7 in Bodenler et al., Molecular Physics $2018^{33}$ ). In this work, we present the instrumental developments of a FFC-MRI system designed to operate between 1 and $2 \mathrm{~T}$ for sample and small animal imaging. The system comprises a resistive solenoidal coil inserted into a $1.5 \mathrm{~T}$ superconductive MRI system. Here, a simple design is shown making use of passive antiparallel power diodes added in series with the high current amplifier so as to increase resistance for low voltages. This design enables to reach large and fast field shifts, while compensating eddy-currents during detection using an additional low power amplifier and reaching enough field stability for imaging. The performances of the system are shown, in terms of stability and field-control capabilities. Thanks to this setup, new methods are proposed to measure both $\mathrm{R}_{1}$ and $\mathrm{R}_{2}$ dispersion profiles, validated against literature data on various dispersive samples. Imaging capabilities to generate dispersive property mapping are shown.

\section{METHODS}

In this section, the FFC-MRI hardware and the methods to characterize the system are first described. The FFC pulse sequences to measure $\mathrm{R}_{1}$ and $\mathrm{R}_{2}$ dispersions are then given. Adaptation to MRI for longitudinal NMR relaxation rate dispersion measurement is finally provided. 


\section{FFC-MRI hardware}

The system comprises an unshielded low-resistance dual-layer solenoidal insert made of silver enclosed into Plexiglass and cooled with perfluorocarbon leaving a 40-mm diameter free-bore (designed and built by Stelar s.r.1, Mede, Italy) (Fig. 1-a). The insert was designed to reach up to 0.48 $\mathrm{T}$ in $4 \mathrm{~ms}$. The manufacturer provided the following additional specifications: resistance less than 0.1 $\Omega$ and a homogeneity better than $100 \mathrm{ppm}$ on a $25-\mathrm{mm}$ diameter sphere. The compact Plexiglass enclosure was $17 \mathrm{~cm}$ in diameter and $30 \mathrm{~cm}$ in length with a total weight of $6 \mathrm{~kg}$. The cooling liquid circulating into the Plexiglass housing was perfluoropolyether (Galden SV110, Solvay) supplied through 10-m long conductive rubber pipes connected to a heat exchanger comprising a plate exchanger with a secondary lost-water circuit and designed to have a cooling capacity of $20 \mathrm{~kW}$. To avoid static charge accumulation, a ground cable was connected between the Faraday cage and the Plexiglass cover. The insert comprised a safety control circuit, featuring internal overheating interlock sensors.

A NMR low-frequency (0.1-125 MHz) pulse sequencer (Apollo, Tecmag, Houston, USA) encompassing one channel for transmission and one channel for reception was used. It was equipped with a gradient waveform synthesizer used to control a high-power current amplifier (Copley 234P04) for large field variations (capable of $300 \mathrm{~A}$ DC and $375 \mathrm{~A}$ for $500 \mathrm{~ms}$ at 50\% duty cycle in current mode, and $23 \mathrm{kVA}$ DC in voltage mode on a load of $0.45 \Omega$ ), and a low current auxiliary amplifier for fine tuning during detection (for eddy-current compensation, model AD822AR, Analog Devices, MA, USA, controlled by an optical coupler ACPL-C87AT, Avago Technologies, USA). Both amplifiers were driven in voltage mode to reduce the risks of electric shocks and potential hardware damage. A non-linear element comprising a pair of anti-parallel diodes and a low-pass filter were inserted at the MRI room filter panel. The low-pass filter ensured radiofrequency cut-off inside the Faraday cage. The diode pair (IRGTI200F06, International Rectifier, USA) limited current fluctuations to obtain a more stable magnetic field for low currents. Only the free-wheel diodes were used by connecting the gate to the emitter. The threshold voltage was $0.4 \mathrm{~V}$ and the maximum continuous current 200 A. The diodes were mounted onto a plate-fin air heat exchanger. For eddycurrent compensation, the auxiliary circuit for controlling the required compensating low current was mounted in parallel to the main power supply, the operational amplifier controlling the low current was in series with a $100 \Omega$ resistor to insulate the main power supply from the auxiliary one (Fig.1c) and to gain in current control precision for low field offsets in the detection range.

The insert was fixed firmly onto the bed of a $1.5 \mathrm{~T}$ clinical MRI system (Fig. 1) (Philips Achieva, Best, The Netherlands) ensuring that the centers coincided (see Bödenler et $a l .{ }^{33}$ for a typical 
integration of a FFC insert in an existing MRI installation). The RF chain comprised an active T/R switch and a preamplifier connected to home-made radiofrequency coils. A 30-mm diameter 50-mm long saddle-shaped volume coil and a form-fitted 20-mm surface coil were constructed ${ }^{39}$. For fieldcycling experiments, the whole setup was used either in a spectroscopic mode independent from the MRI scanner (then the Apollo sequencer was also used for RF transmission and NMR signal collection), or in an imaging mode in which the modified MRI sequences triggered the FFC sequencer (then used only to control both power amplifiers). In spectroscopic mode, a 2-kW linear pulse RF amplifier was used (ENI MRI-2000), while in imaging mode, the default MRI RF amplifier was used. The pulse sequencer software NTNMR was used to define the events in various pulse sequences. The latter comprises automation capabilities that were interfaced using Matlab (The MathWorks, Natick, MA, USA) to automate the parameter settings, data acquisition, calibrations and analysis. Pulse sequences combining FFC and compensation waveforms, RF pulses as well as acquisition periods were implemented to calibrate and to compensate eddy currents, as described below.

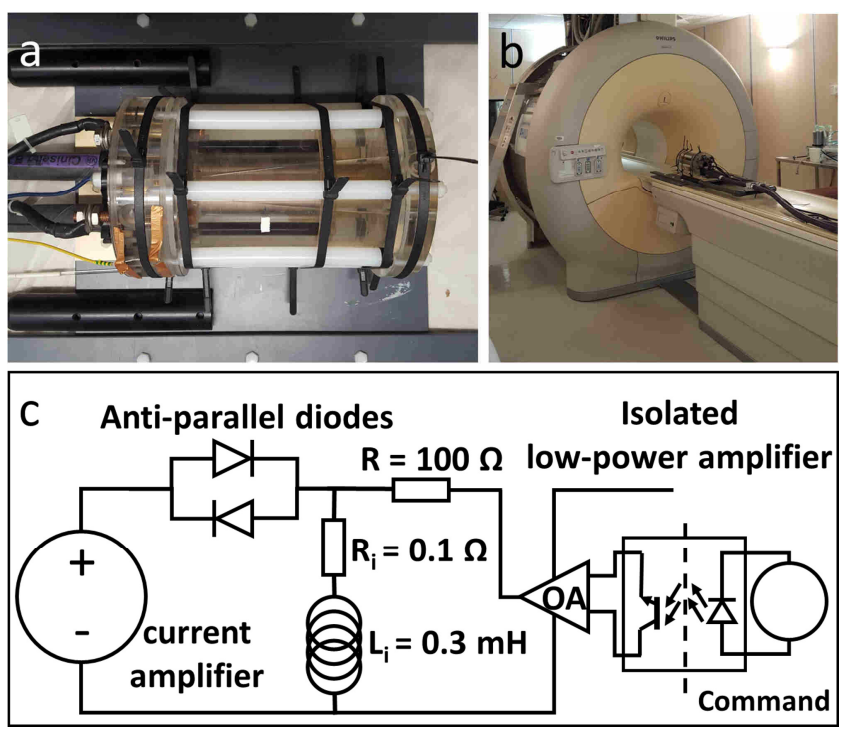

Figure 1: FFC-MRI system integration $(a-b)$ and schematic electrical drawings of the system mounted with the power diodes and the compensation low power amplifier (c). (a) The 40-mm clear bore and its Plexiglass housing set on a specific holder adapted to the MRI bed ensured tight fixation of the insert. (b) general view of the MRI room with the insert set on the MRI bed. (c) The insert is driven by two amplifiers that are passively separated. In high power mode, the anti-parallel diodes resistance is negligible and the current flows mainly into the insert because the resistance $R_{i}$ is small as compared to $R$. In low power mode, the current can be controlled precisely using a low power amplifier in combination with a high output resistance $R$. $L_{i}$ denotes the insert inductance. 


\section{System calibration methods}

For high $\mathrm{B}_{0}$ field offsets, the Copley amplifier voltage and current monitors were used to calibrate the system response and check the effective waveforms. Their measure was done using either a digital oscilloscope, or a data acquisition board (BNC 2110, National Instruments) synchronized to the Tecmag pulse sequencer and driven using Matlab. For small $\mathrm{B}_{0}$ offset values, the precision of the monitors was not sufficient, and NMR frequency was used as a monitor of the effective current. This was possible for frequency offsets within the range of the receiver chain as detailed below. We describe here the methods that were applied to precisely measure the NMR frequency in a variety of situations in this range, to calibrate the system response to a step voltage command, to prescribe and check the $\mathrm{B}_{0}$ offset waveforms, to measure eddy-currents and the system stability, and to measure the FFC insert homogeneity.

To precisely measure the mean NMR frequencies over a sample in real-time, two strategies were followed. The first one is based on the acquisition of free induction decay (FID) signals after a short $90^{\circ} \mathrm{RF}$ pulse (Fig. 2). The receiver bandwidth was set to the maximum possible value (1 MHz), so that variations in the range $\pm 500 \mathrm{kHz}$ NMR frequency could be probed. A reference FID acquisition was first performed with the amplifier disabled providing a measure of a reference phase evolution. Then, a FID was acquired in a different situation, e.g, the amplifier enabled or applying a waveform. The processing was performed using Matlab. After subtraction of the reference phase, the phase of the FID was first unwrapped in time before being time differentiated to provide an instantaneous frequency measurement. To reduce the noise on the instantaneous frequency measurement as a result of the large acquisition bandwidth, it was chosen to low-pass filter it numerically, which is justified by the fact that the current variations, and consequently the NMR frequency variations are intrinsically filtered by the FFC insert. A Butterworth filter of order 4 with a 2-to-10 kHz bandwidth was used. The precision of such measurement technique can be estimated from the SNR. Indeed, for a given acquisition bandwidth $\left(\mathrm{BW}_{\mathrm{acq}}=1 \mathrm{MHz}\right)$, the standard deviation of the instantaneous phase measurement can be approximated by $1 / \mathrm{SNR}^{40}$. The process of differentiating the phase to estimate instantaneous frequency and low-pass filtering it with a Butterworth filter leads to a standard deviation of frequency well approximated by $\mathrm{SNR}^{-1} \pi^{-1} \mathrm{BW}_{\mathrm{acq}}{ }^{-1 / 2} \mathrm{BW}_{\text {filter }}{ }^{3 / 2}$, for $\mathrm{BW}_{\text {filter }}<0.2 \mathrm{BW}_{\mathrm{acq}}$, such that, for example, an instantaneous SNR of 30 at a 1-MHz bandwidth provides a 1-Hz precision on the NMR frequency if filtered with a $2 \mathrm{kHz}$ bandwidth, and a $10-\mathrm{Hz}$ precision with a $10-\mathrm{kHz}$ bandwidth. Due to the time decay of the FID signal, the NMR frequency could typically be measured over a limited duration of $32 \mathrm{~ms}$ using this strategy. To cover longer temporal windows, such as for eddy-current measurement after the application of a $B_{0}$ offset pulse (Fig. 3-a), experiments were 
repeated by varying the delay between the pulse and the single-FID sequence with a sufficiently long repetition time TR to ensure that magnetization recovers and eddy-currents vanish. To perform faster frequency measurements in a continuous manner (i.e. requiring a single experiment, while measuring for temporal windows longer than the FID signal duration), a second strategy was followed involving the fast repetition of small RF pulses (Fig. 3-b).
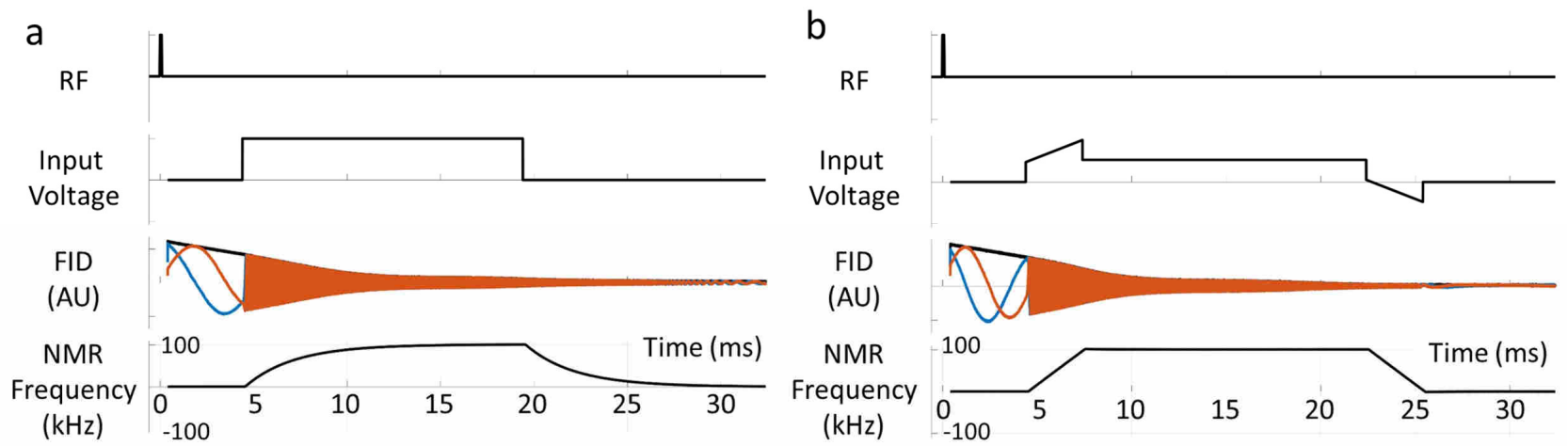

Figure 2: NMR sequences used for the calibration of the system and the waveforms. The amplifier is operated in voltage mode, regulating internally the output voltage applied over the insert, without feedback on the current waveform. (a) FIDs (real, imaginary and magnitude shown) were collected during the application of a 15-ms step voltage command to obtain the insert current response (NMR frequency calculated from the filtered signal phase) and extract the characteristic time; (b) the command needed to obtain a prescribed current waveform (here a trapezoid with 3 ms ramp time and $15 \mathrm{~ms}$ plateau) is then applied. Data presented here for a $100 \mathrm{kHz}$ NMR frequency plateau, phase filtered at $B W_{\text {filter }}=10 \mathrm{kHz}$, and signal acquired during $32 \mathrm{~ms}$. 


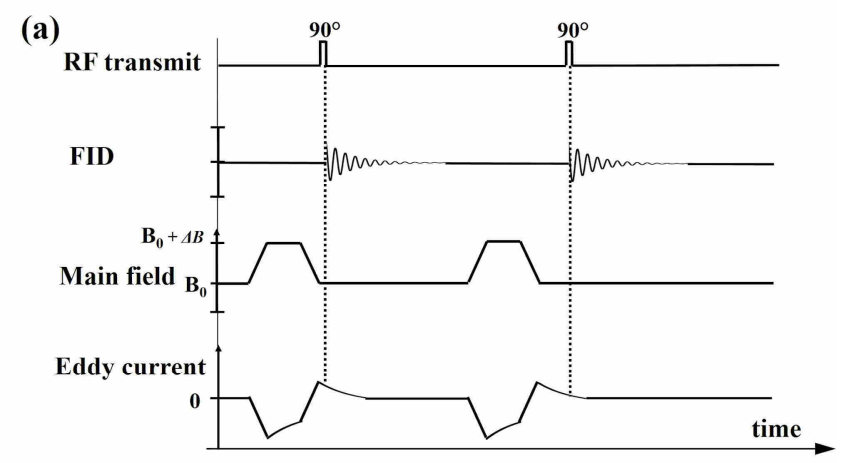

(b)

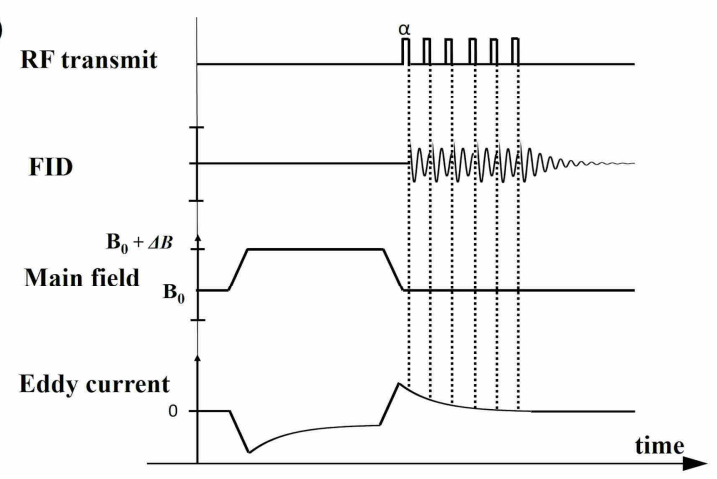

Figure 3: NMR sequences used for the measurement of the magnetic field perturbation due to eddycurrents after a field offset pulse: (a) FIDs were collected for several delays after the end of the offset field pulse, after a $90^{\circ} \mathrm{RF}$ pulse and a typical 3-s repetition time between each measurement; $(b) a$ train of FIDs (typically $10^{\circ}$ flip angle, 4-ms repetition time) was collected after a single field-offset pulse; with this approach one could rapidly follow the frequency with the objective to apply iterative corrections of the auxiliary current to compensate for it.

\section{Stability, efficiency, eddy-current characterization and compensation}

Magnetic field stability and calibration were evaluated through NMR measurements acquired using the system in its spectroscopic mode on a $3-\mathrm{cm}$ diameter spherical water phantom placed into the saddle volume coil. With FIDs, NMR frequency and phase were measured to characterize the stability of the field close to zero current (when phase stability is the most critical). Measurements with the power supply enabled (but without any offset field applied) were repeated with a long repetition time to ensure recovery to thermal equilibrium from one excitation to the next.

The amplifier was operated in voltage mode, regulating internally the output voltage applied over the insert, without feedback on the current. To calibrate the response of the system (produced $\mathrm{B}_{0}$ field offset frequency versus voltage) and to check the shape reproducibility for a prescribed frequency offset, several experiments targeting different offsets were reproduced 16 times for different voltage step commands (Fig.2-a). The transient state of the LR circuit constituted by the insert coil and the 
cables was used to estimate the characteristic time by fitting the NMR frequency-versus-time curve to an exponential decay. To obtain a targeted current waveform $i(t)$ (such as a trapezoid in Fig.2-b), the command (input voltage to the Copley amplifier) was set proportional to the required output voltage $u(t)=R_{i} i(t)+L_{i} \frac{d i}{d t}(t)$, where $R_{i}$ denotes the resistance of the insert and the cables, and $L_{i}$ the insert inductance.

Eddy-currents were characterized by measuring the NMR signal frequency after the end of a $\mathrm{B}_{0}$ offset pulse, first by repeating the measurement with a $90^{\circ} \mathrm{RF}$ pulse for several delays (Fig.3-a). Delays between the end of the $\mathrm{B}_{0}$ offset pulse and the RF pulse were incremented by step of 10 to $25 \mathrm{~ms}$. A long repetition time of typically $3 \mathrm{~s}$ was chosen to avoid residual eddy currents from one repetition to the next. Due to this long delay, a detailed measurement of the eddy-current decay corresponded to an acquisition time of few minutes. The faster method (Fig 3-b) was also applied with a $10^{\circ}$ flip angle, a 4-ms acquisition time ( $\mathrm{TR}=4.2 \mathrm{~ms}$ ) and 240 repetitions requiring a one-shot experiment lasting about $1 \mathrm{~s}$. Based on this measurement, the low-amplifier command was iteratively adapted to counteract the eddy-currents and reduce the frequency shifts.

To measure the FFC insert homogeneity, the system was used in imaging mode. A 50-mL BD Falcon tube (27-mm diameter 114-mm long cylinder) filled with water was placed into the volume coil. Multi-echo 3D gradient echo MRI pulse sequences were applied with the following parameters: fieldof-view FOV = 128x64x64 $\mathrm{mm}^{3}, 1-\mathrm{mm}$ isotropic acquisition voxel size interpolated to $0.5 \mathrm{~mm}$, pixel bandwidth $\mathrm{BW}_{\text {pix }}=893 \mathrm{~Hz}$, echo time $\mathrm{TE}=2.06 \mathrm{~ms}, 16$ echoes with an inter-echo time of $1.62 \mathrm{~ms}$, and TR $=37 \mathrm{~ms}$ for a total acquisition time of $2 \mathrm{~min}$. This sequence was applied with different stationary currents applied into the insert (-32 to $32 \mathrm{~mA})$. The k-space signals were numerically corrected along the readout direction (multiplied by a complex exponential $\exp (-2 i \pi f t)$ to compensate for the central NMR frequency $f$ which was different for each applied current), frequency maps calculated by fitting voxel-wise the frequency from the phase evolution as a function of echo time ${ }^{41}$, and finally subtracted to isolate the effects of the insert inhomogeneity.

\section{FFC sequences and methods for dispersion quantification}

$R_{1} N M R D$

To evaluate the capability of the system to measure longitudinal NMRD profiles, an inversionrecovery sequence was implemented in spectroscopic mode. For these experiments, the antiparallel diodes were not used and the Copley amplifier was disabled during RF pulses and acquisition periods. 
The sequence (see Fig. 7-a in Bodenler et al., Molecular Physics $2018^{33}$ for a scheme of the sequence) was repeated with a time ranging from 1 to $10 \mathrm{~s}$ that was each time sufficiently long to recover most of the longitudinal magnetization, and was composed of a $180^{\circ}$ inversion pulse, an evolution time during which a $\mathrm{B}_{0}$ offset pulse was applied, a $90^{\circ}$ excitation and a FID (16 ms acquisition time with a $125 \mathrm{kHz}$ bandwidth). Hard RF pulses were used (200 $\mu$ s duration for the $180^{\circ}$ pulse and $100 \mu \mathrm{s}$ for the $90^{\circ}$ pulse). The evolution period comprised $10 \mathrm{~ms}$ before and $13 \mathrm{~ms}$ after the $\mathrm{B}_{0}$ offset pulse. At each repetition, the amplitude and duration of a voltage step command were changed to produce a $\mathrm{B}_{0}$ offset ranging from -18 to $18 \mathrm{MHz}$ during an application time ranging from 0 to $3.2 \mathrm{~s}$. The expected power dissipated in the insert for each waveform was estimated beforehand, and only the waveforms generating less than $1.5 \mathrm{~kJ}$ heat dissipated in the insert resistance were applied. This condition permitted, for example, to apply evolution shifts of $18 \mathrm{MHz}$ up to $160 \mathrm{~ms}, 16 \mathrm{MHz}$ up to $200 \mathrm{~ms}, 10$ $\mathrm{MHz}$ up to $500 \mathrm{~ms}, 7 \mathrm{MHz}$ up to $1 \mathrm{~s}$, and $4 \mathrm{MHz}$ up to $3.2 \mathrm{~s}$.

To recover the NMRD profile, data were processed offline. The first pre-processing step aimed at extracting the signed FID amplitude. Each FID was rephased by a reference phase corresponding to the low pass filtered $(2.5 \mathrm{kHz})$ FID with the longest inversion time and without $\mathrm{B}_{0}$ offset. The real part of the average complex signal of the first $1 \mathrm{~ms}$ of each FID was then calculated. The FID amplitude was then fitted to a standard longitudinal relaxation model with exponential decays encompassing the sequence timings and which parameters to be fitted were the thermal equilibrium magnetization signal at $1.5 \mathrm{~T} \mathrm{M}_{0}$, the inverted magnetization signal $\mathrm{M}_{\mathrm{i}}$, and the relaxation rates $\mathrm{R}_{1}(\Delta B)$ at each probed field offset $\Delta B$, in particular accounting for the relaxation to a shifted thermal equilibrium during the applied field offsets. The relaxation rates were then fitted using a first order polynomial as $\mathrm{R}_{1}(\Delta B)=\mathrm{R}_{1,0}+\beta_{1} \times \Delta B$ where $\mathrm{R}_{1,0}$, denotes the longitudinal relaxation rate at $\mathrm{B}_{0}=$ $1.5 \mathrm{~T}, \beta_{1}$ the slope of the NMRD profile and $\Delta B$ the field offset. Validation experiments were performed at $293 \mathrm{~K}$ using MRI contrast agent solutions diluted in water and placed into a 30-mm diameter sphere centered into the volume coil. Water, non-dispersive ${ }^{42} 0.166,0.333$ and $1 \mathrm{mM}$ gadoterate meglumine solutions (Dotarem, Guerbet, France) (referred to as Gd-DOTA) and dispersive (from 20 to $200 \mu \mathrm{M}$ by steps of $20 \mu \mathrm{M}$ ) ultra-small particles of iron oxides solutions (CL30Q02-02 Molday ION, BioPAL, MA, USA) (stable aqueous solution of superparamagnetic 30-nm dextran-coated magnetite particles with 8-nm mean core size referred to as USPIO here) were characterized. 


\section{$R_{2} N M R D$}

The quantification of proton transverse relaxation dispersion also presents an interest to investigate microscopic systems. Only few measurements with fast field cycling relaxometry are reported in the literature, and in limited range (see for example early works in the range 0-2 $\mathrm{MHz}^{43,44}$, and more recently up to $20 \mathrm{MHz}^{45}$ ). To validate the possibility to quantify transverse dispersion with the FFC setup around $63.8 \mathrm{MHz}$, two well characterized systems of interest for biomedical imaging were considered. Experiments were performed at room temperature $(293 \mathrm{~K})$. The first sample consisted in $13 \mathrm{mM}$ Gd-DOTA diluted in water. This gadolinium chelate does not show significant transverse relaxation dispersion at $1.5 \mathrm{~T}^{42}$. The second sample consisted of a ferritin solution (horse spleen ferritin, F4503, Sigma Aldrich) at $170 \mathrm{mM}$, for which a strong linear increase of transverse relaxation rate has been reported ${ }^{14}$. The solutions were placed into an $18-\mathrm{mm}$ diameter spherical container centered into the volume coil.

The measurement of transverse relaxation dispersion was done here using the antiparallel diodes. A FFC spin-echo sequence depicted in Fig. 4 was applied. Echo time was $29.57 \mathrm{~ms}$ and repetition time $1.45 \mathrm{~s}$. Identical $\mathrm{B}_{0}$ offset pulses were inserted before and after the $180^{\circ}$ refocusing pulses in a dual echo sequence. The voltage commands consisted in 2-ms long steps with different amplitudes, the first one applied $1.36 \mathrm{~ms}$ after the $90^{\circ}$ pulse (100 $\mu$ s duration), the second and fourth one $0.3 \mathrm{~ms}$ after the $180^{\circ}$ pulses $(200 \mu \mathrm{s})$, and the third one $1.36 \mathrm{~ms}$ after the first echo time. The actual current waveforms provided by the current monitor were acquired simultaneously to the NMR signals. The acquisition of the FID as well of the echoes was continuous starting $350 \mu$ s after the $90^{\circ}$ pulse, blanking the receiver during the $180^{\circ}$ pulses. An acquisition performed without $\mathrm{B}_{0}$ offset pulse was used to extract a reference phase that was removed from all other acquisitions. The average of the FID signal magnitude from the first $100 \mu \mathrm{s}$ was used to normalize the acquisitions (by the thermal equilibrium value at $1.5 \mathrm{~T}$ ). The echo magnitudes were calculated as the average normalized signal over a 1-ms window around the echo times. For each spin-echo acquisition, the field offset $\left(B(t)-B_{0}\right)$ was measured continuously using the data acquisition board and its area was calculated accordingly (noted $A$ in the following for the integral between 0 and the echo time, given in T.s). If the area of one of the four sections varied by more than $2 \times 10^{-6}$ T.s of the average areas, the measurement was discarded. This cut-off was chosen empirically after performing preliminary experiments that purposely varied the difference between two consecutive waveforms, selecting a value ensuring that spin-echo refocusing was good enough, as assessed by an echo magnitude variation of less than $1 \%$ over the tested range of areas. The normalized echo magnitude was then studied as a function of $A$ and fitted in the least squares sense to the following signal equation: 
$\exp \left(-R_{2,0} T E \quad\right) \exp \left(-\beta_{2} A\right)$ (see appendix). The first exponential is the usual transverse signal decay at $1.5 \mathrm{~T}$ characterized by the relaxation rate $\mathrm{R}_{2,0}$, the second one accounts for the variation of the transverse relaxation rates assuming $\mathrm{R}_{2}(B(t))=\mathrm{R}_{2,0}+\beta_{2} \times\left(B(t)-B_{0}\right)$. The residual between the model and the measured signal was then used to estimate the standard deviation on the estimated parameters $\mathrm{R}_{2,0}$ and $\beta_{2}$ using Monte-Carlo simulations (standard deviation over 1000 fits of simulated signals with the addition of random noise).

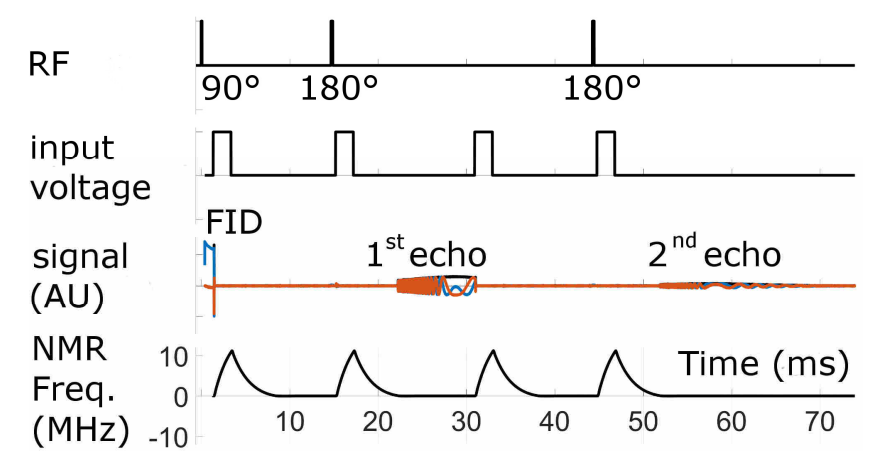

Figure 4: Pulse sequence applied for $T_{2}$-dispersion measurement for the maximum area applied (1.41 $\times 10^{-3}$ T.s, i.e. corresponding to an area of $0.353 \mathrm{~T}$ for $4 \mathrm{~ms}$ ). The signal cancels when the NMR frequency goes outside the receive bandwidth or when the phase dispersion is too large. Spin echoes are formed when the areas of the Bo offset pulses balance. The initial FID magnitude is used as a reference to normalize the echo magnitude.

\section{$\underline{\text { R1-dispersion mapping }}$}

An MRI protocol was defined for imaging at $1.5 \mathrm{~T}$ and for FFC relaxometric mapping. The protocol comprised first a localisation scan performed at $1.5 \mathrm{~T}$ targeting $0.5 \mathrm{~mm}$ isotropic voxel size, before the acquisition of a single slice with a 2D FFC inversion recovery multiple spin-echo sequence. The system was used without the anti-parallel diodes as the sequence timings allowed including enabling and disabling periods for the amplifier. Indeed, the current amplifier took $350 \mathrm{~ms}$ to be ready to deliver the requested power, allowing to apply RF inversion pulses right before the effective activation, and the $\mathrm{B}_{0}$ field shift rapidly after it. After the field shift, the amplifier outputs could be isolated in $2 \mathrm{~ms}$ before the application of the $90^{\circ} \mathrm{RF}$ pulse of the spin-echo sequence, during which the fast eddy-current compensation technique described above was used. The following imaging parameters were used: readout field-of-view FOV $=70 \mathrm{~mm}$, phase field-of-view $=32 \mathrm{~mm}, 0.5 \mathrm{~mm}$ isotropic acquisition voxel size, slice thickness $2.5 \mathrm{~mm}$, pixel bandwidth $\mathrm{BW}_{\text {pix }}=200 \mathrm{~Hz}, 2$ signal accumulations, echo time $\mathrm{TE}=13.5 \mathrm{~ms}$, echo spacing $13.5 \mathrm{~ms}, 8$ echos, and TR $=2000 \mathrm{~ms}$ for a total acquisition time of $4.3 \mathrm{~min}$. The same slice was acquired in five different conditions: three 
acquisitions used the same TI of $540 \mathrm{~ms}(10 \mathrm{~ms}$ ramp time and a $500 \mathrm{~ms}$ plateau) but different relaxation fields (1.34 T, $1.5 \mathrm{~T}$ and $1.66 \mathrm{~T}$, images will be referred to as $\mathrm{M}_{-}, \mathrm{M}$ and $\mathrm{M}_{+}$, respectively), an acquisition (noted $\mathrm{M}_{\text {inv }}$ ) was done with a minimum inversion time $(10 \mathrm{~ms})$ at $1.5 \mathrm{~T}$ and an acquisition without any inversion pulse $\mathrm{M}_{\text {noinv }}$ at $1.5 \mathrm{~T}$. The complex images were first phase corrected using the phase of a low pass-filtered version of $\mathrm{M}_{\text {noinv }}$. The real part of the images were then fitted voxel-wise to the Bloch equations assuming a linear approximation of the NMRD profile. The thermal equilibrium magnetization at $1.5 \mathrm{~T} \mathrm{M}_{0}$ was expressed as a function of $\mathrm{M}_{\text {noinv }}$ assuming a recovery time of TR-8TE, the inverted magnetization $\mathrm{M}_{\mathrm{i}}$ after a recovery time of TR-TI-8TE was expressed as a function of $M_{i n v}$ and $M_{0}$. Images $M_{-}, M_{\text {and }} M_{+}$theoretical evolution were finally expressed as a function of $M_{i n v}, M_{\text {noinv }}$, the relaxation rate $R_{1,0}$ at $1.5 \mathrm{~T}$ and the slope of the NMRD profile $\beta_{1}$ at 1.5 $\mathrm{T}$. The data were fitted to the model using a non-linear least squares Gauss-Newton iterative algorithm with 1000 iterations after initialisation of $R_{1,0}$ maps to the positive and small value of $0.2 \mathrm{~s}^{-1}$ and $\beta_{1}$ maps to $0 \mathrm{~s}^{-1} \mathrm{~T}^{-1}$ (no dispersion).

To validate the ability of the sequence to map $\mathrm{R}_{1}$ dispersion around $1.5 \mathrm{~T}$ (i.e. $\beta_{1}$ ) with this protocol, imaging experiments were performed at $293 \mathrm{~K}$ on 4 samples placed into 4-mm diameter cylindrical tubes containing water, Gd-DOTA, and USPIO in water. Concentrations were chosen so that to obtain $\mathrm{R}_{1,0}$ values close to $1 \mathrm{~s}^{-1}$, as measured in spectroscopic mode to be in the range of values encountered in vivo (between $45-75 \mu \mathrm{M}$ for the USPIO samples). RF transmit and receive was ensured by the surface coil mounted on top of the tubes.

\section{RESULTS}

\section{System calibration}

The measured insert resistance $(102.7 \pm 0.6 \mathrm{~m} \Omega)$ was deduced from the voltage measured over the insert while imposing a known current. With the additional 10-m long power cables, the total resistance was $R_{i}=115 \pm 1 \mathrm{~m} \Omega$. Assuming an inductance-resistance low-pass system, the coil inductance $\left(\mathrm{L}_{\mathrm{i}}=302 \pm 3 \mu \mathrm{H}\right)$ was deduced from the time constant $(2.625 \pm 0.001 \mathrm{~ms})$ after applying a voltage step over the insert, measuring the NMR frequency temporal evolution of a water sample and fitting it with an exponential decay (Fig.2-a). The coil efficiency (1.387 $\pm 0.004 \mathrm{mT} / \mathrm{A})$ was estimated from the NMR frequency plateau obtained for various voltage steps lower than 8 A to remain in the bandwidth of the NMR radiofrequency receiver coils, and from the current monitor for larger currents (Fig. 5). Without the antiparallel diodes, the current plateau (or equivalently the NMR frequency) was linear with the voltage command. With the antiparallel diodes, the system response indicated a slightly increased resistance of $120 \mathrm{~m} \Omega$ for high voltage (threshold voltage higher than $0.4 \mathrm{~V}$ ), while 
it was increased to $6.4 \Omega$ for lower voltages. From the repeated measurements of the same 16 step voltages from -100 to $100 \mathrm{kHz}$, the instantaneous reproducibility of the NMR frequency curves was $\pm 50 \mathrm{~Hz}$. Using these calibration, it was then possible to define the voltage command that resulted in a predefined current waveform, as displayed in Fig.2-b with a trapezoidal waveform.

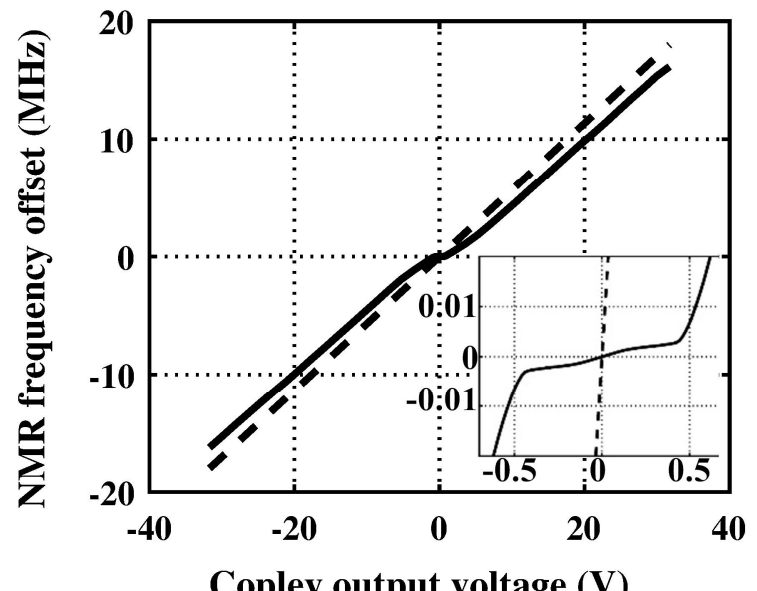

Figure 5: NMR frequency offset as a function of Copley output voltage after the establishment of the steady-state current without the power diodes (dashed line) and with the power diodes in series with the insert (solid line): the NMR frequency offset was computed from the current monitor for voltage amplitudes higher than $1 \mathrm{~V}$, and in the low voltage range from NMR frequency measurements (inserted graph).

\section{Stability of the magnetic field}

Fig. 6 shows NMR phase and frequency measurements obtained with the Copley amplifier enabled but when zero current should be delivered to the insert coil. Several FIDs were acquired and data for only three of them are shown for clarity. The observation time of $20 \mathrm{~ms}$ corresponds to typical encoding durations needed for consistent MRI acquisitions. When the Copley amplifier was directly connected to the insert (Fig.6-a), phase excursion over $20 \mathrm{~ms}$ or from one measurement to the next could be larger than 3 rad. By contrast, with the diode pair connected (Fig.6-b), the phase change did not exceed $0.1 \mathrm{rad}$. Similarly the frequency offsets could change by $200 \mathrm{~Hz}$ over $20 \mathrm{~ms}$ from one measurement to the next without the diode pair (Fig.6-c), but remained below $7 \mathrm{~Hz}$ (mean over the standard deviation of several measurements) with the diode pair connected (Fig.6-d). The instability of the NMR frequency by $200 \mathrm{~Hz}$ corresponds to a current noise of about $3 \mathrm{~mA}$ in the insert coil, which can thus be reduced below $0.1 \mathrm{~mA}$ with the diodes connected. Typical intensities for the offset field were in the range of $0.5 \mathrm{~T}$, corresponding to an NMR frequency shift around $20 \mathrm{MHz}$. The current in the insert was thus controlled with the required $10^{-6}$ relative accuracy during detection with the diode pair. 
(a)

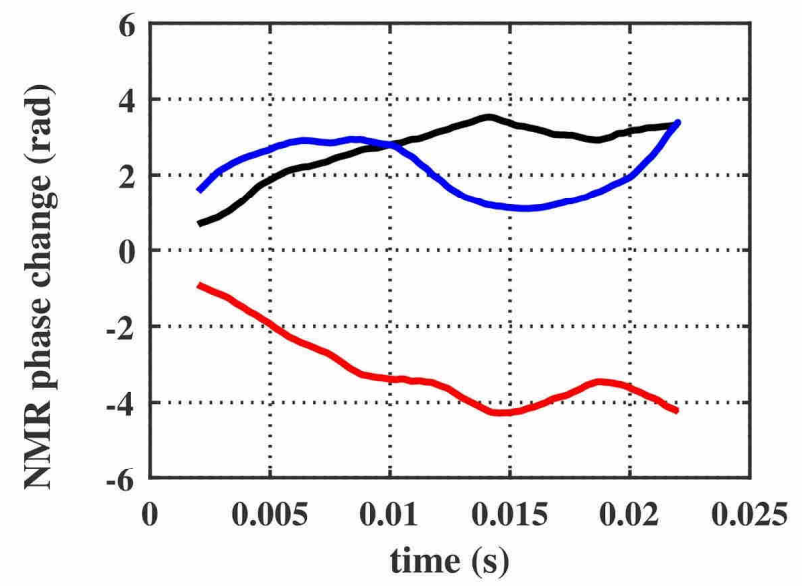

(c)

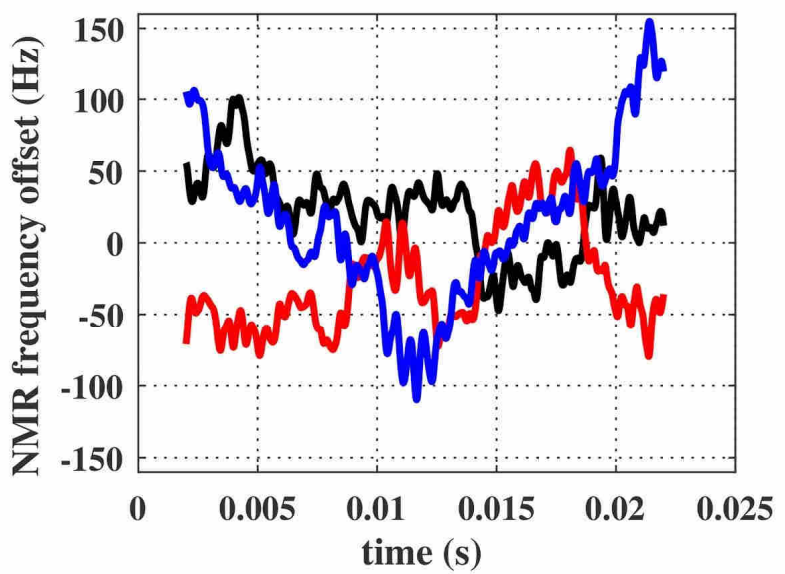

(b)

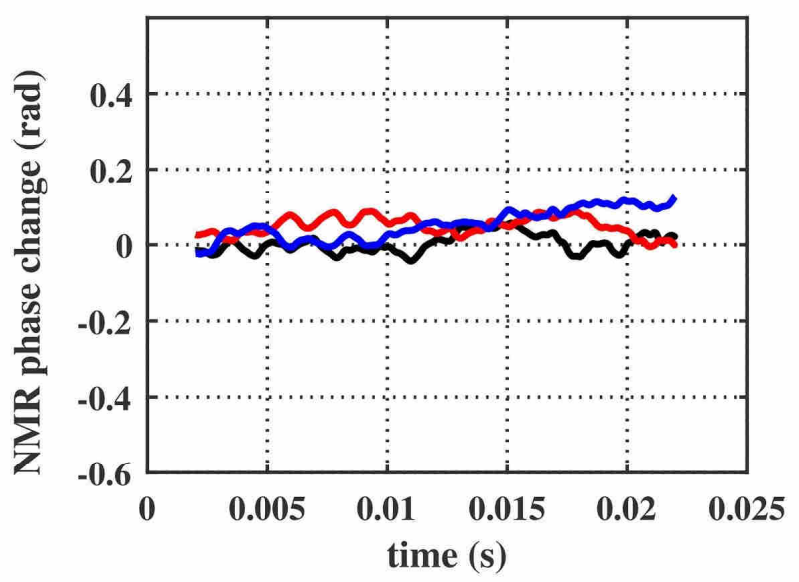

(d)

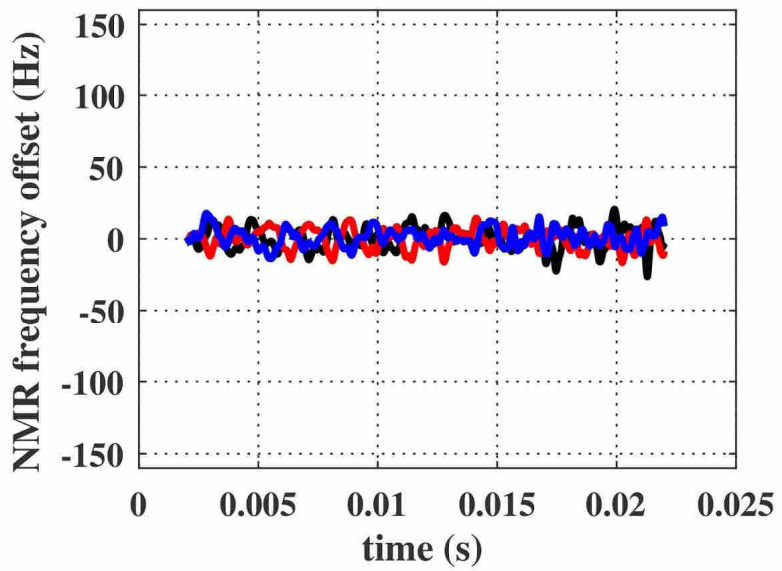

Figure 6: NMR phase changes $(a, b)$ measured from successive FIDs measured in a $3 \mathrm{~cm}$ water sphere in the volume coil, and NMR frequency offsets $(b, d)$ computed from the phase data: $(a),(c)$ the Copley amplifier was directly connected to the insert, $(b),(d)$ the diode pair was inserted between the Copley amplifier and the insert.

\section{Eddy-current measurement and compensation}

Fig. 7-a shows the NMR frequency shift after $500 \mathrm{~ms} \mathrm{~B}_{0}$ offset pulses. Data were collected for 20 different delays starting $12 \mathrm{~ms}$ after the end of the pulses and concatenated to cover $500 \mathrm{~ms}$ frequency shifts (method shown in Fig 3-a). Each pulse was repeated every $3 \mathrm{~s}$. Measurements performed with different intensities of the offset pulse showed that the eddy-currents were proportional to the $\mathrm{B}_{0}$ field offset. A mono-exponential fit to these data gave an extrapolated amplitude after the pulse of 148 $\mathrm{Hz} / \mathrm{MHz}$ and a time constant of $104 \mathrm{~ms}$, but did not model the effects entirely (residual standard deviation $14.5 \mathrm{~Hz}$ ). A dual exponential fit modelled better the effects (residual standard deviation $10.2 \mathrm{~Hz}$ ) and gave amplitudes of 52 and $106 \mathrm{~Hz} / \mathrm{MHz}$ with time constants respectively of 170 and 69 ms. 
Fig. 7-b displays NMR frequency offsets obtained without eddy-current compensation using the method described in Fig 4-b (RF pulse train). It provides similar measurement of the eddy current decay demonstrating that a fast measurement after a single field offset pulse can be performed ( $141 \mathrm{~Hz} / \mathrm{MHz}$ and a time constant of $93 \mathrm{~ms}$ for a single exponential decay model, 122 and $44 \mathrm{~Hz} / \mathrm{MHz}$ with time constants respectively of 111 and $19 \mathrm{~ms}$ for a dual exponential decay). To compensate the eddy currents for potentially different duration, ramps, or repetition time, a fast adjustment method was set up by iteratively updating the voltage command of the low power amplifier to cancel the measured eddy-current shifts (Fig. 7-b). For a prescribed waveform, compensation of the eddycurrents could be obtained after only few iterations, with a cancelation better than $50 \mathrm{~Hz}$ for a typical temporal window starting $12 \mathrm{~ms}$ after the field shift and up to $500 \mathrm{~ms}$ after the application of this 500$\mathrm{ms}, 7-\mathrm{MHz}$ pulse. This pulse is then applied in the FFC-IR imaging sequence with this correction.

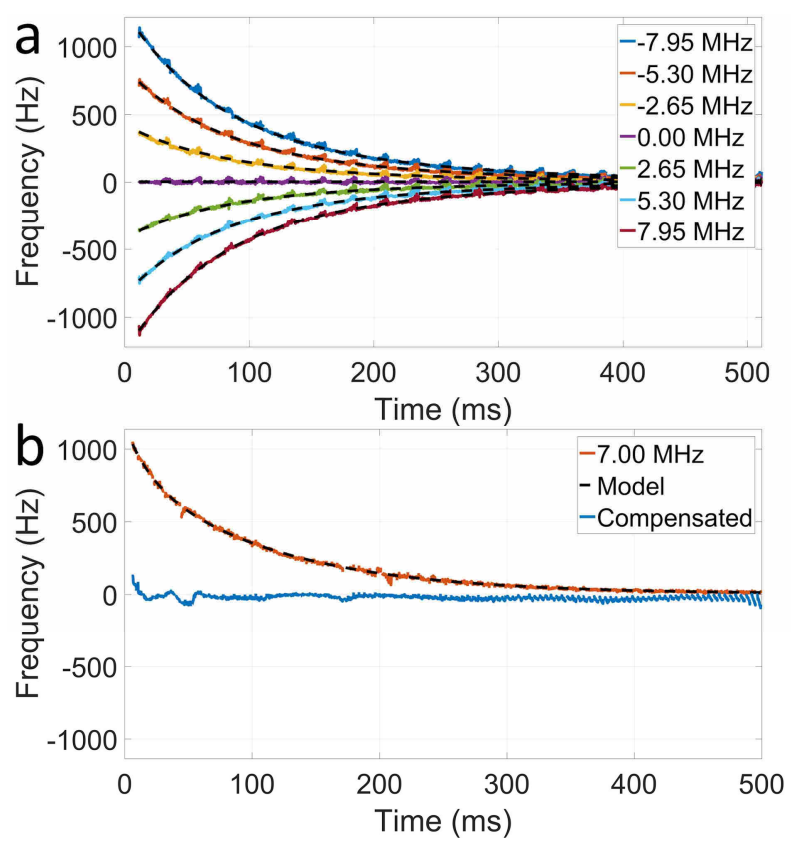

Figure 7: Eddy currents detected as NMR frequency shifts after a Bo field offset pulses : (a) 500-ms field offset pulses between -7.95 and $7.95 \mathrm{MHz}$ by 2.95-MHz steps: data collected as described in Fig 3-a for 20 delays (every $25 \mathrm{~ms}$, data concatenated) ). The superimposed dashed lines correspond to the simultaneous fit of all data to a dual exponential decay model; (b) Eddy currents measured with the fast method for a field offset pulse (7-MHz, 10-ms ramp times, 500-ms plateau time) Data collected as described in Fig 3-b after a single Bo field offset pulse; efficient compensation of the eddy current (EC) was visible when the compensation current in the insert coil provided by the auxiliary power supply was applied. 


\section{Insert homogeneity}

Fig. 8 presents coronal and axial views of the insert homogeneity measured on the 27-mm diameter cylindrical tube. Twenty-five millimeter circular ROIs drawn in the coronal and axial plane in the most homogeneous central region provided a standard deviation of about 290 and 166 ppm, respectively, indicating a higher homogeneity in the axial plane as compared to the insert axis direction. As can be seen on the profile drawn along the axis direction, the field is more homogeneous at the center (with values of $+400 \mathrm{ppm}$ as compared to the average value used as a reference), and it degrades rapidly with the distance to the center ranging roughly $-400 \mathrm{ppm}$ at position -11 and +14 $\mathrm{mm}$. The most homogeneous $25-\mathrm{mm}$ diameter sphere provided a $268 \mathrm{ppm}$ standard deviation of field (representing $0.113 \mathrm{mT}$ for the maximum value applied here of $0.423 \mathrm{~T}$ ), and a difference of 2473 ppm between extrema in the VOI. Inside a 18-mm diameter sphere, a 147 ppm standard deviation of field, and a difference of 1077 ppm between extrema were estimated

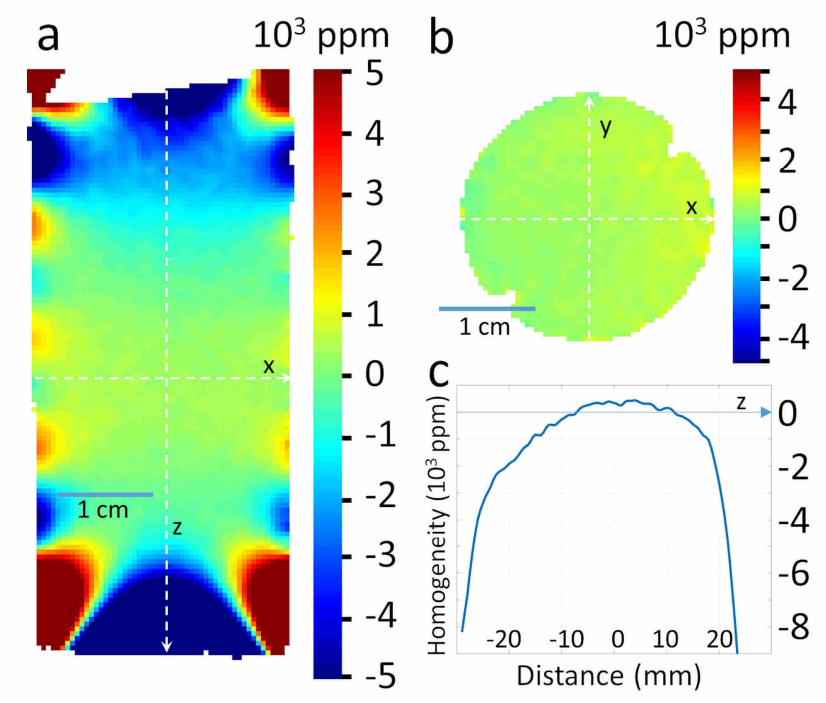

Figure 8: Insert homogeneity (in ppm) in the coronal (a) and axial (b) imaging planes measured on a 27-mm diameter cylindrical tube. As expected, the insert is more homogeneous in the transverse direction as compared to the longitudinal one. The profile along $z$, the insert axis $(c)$ indicates a rapid decrease of the field with the distance to the center. 


\section{$\underline{R_{1} \text { and } R_{2} \text { NMRD measurements }}$}

All the measurements were performed at room temperature (regulated between 293 and $297 \mathrm{~K}$, verified with a thermometer for each experiment and provided with an estimated $1 \mathrm{~K}$ precision). As expected, magnetization recovery after inversion depends on the field offset applied in very different ways for non-dispersive and dispersive samples (Fig. 9-a and b, respectively), enabling to estimate the NMRD profile in the range 1.08 to $1.92 \mathrm{~T}$ (Fig. 9-c and d). The recovery curves modelled well the measured data, with very different recovery trends for dispersive versus non-dispersive samples, combining relaxation and polarization effects. Indeed, long inversion times are displaying the increased polarization, while shorter inversion times (on the order of $T_{1,0}$ ) are more sensitive to dispersion. The NMRD profile for Gd-DOTA is well approximated by a first order polynomial, while a small curvature can be seen for USPIO for large field offsets. For all samples, the NMRD profiles were well modelled by a first order polynomial in the range $\Delta B= \pm 0.23 \mathrm{~T}$, the corresponding $\mathrm{R}_{1,0}$ and $\beta_{1}$ parameters are given in Table 1 . Water was found with a small $\mathbf{R}_{10}$ and a negligible dispersion over this range as expected. Fitting the data as a function of contrast agent concentration provided relaxivities of $\mathrm{r}_{10}=4.31 \mathrm{mM}^{-1} \mathrm{~s}^{-1}$ and $\mathrm{r}_{10}=14.4 \mathrm{mM}^{-1} \mathrm{~s}^{-1}$, and relaxivity slopes of -0.282 and $-9.14 \mathrm{mM}^{-}$ ${ }^{1} \mathrm{~s}^{-1} \mathrm{~T}^{-1}$ for Gd-DOTA and USPIO, respectively, confirming the expected very strong $\mathrm{R}_{1}$-dispersion of USPIOs around $1.5 \mathrm{~T}$ in addition to its larger relaxivity. 

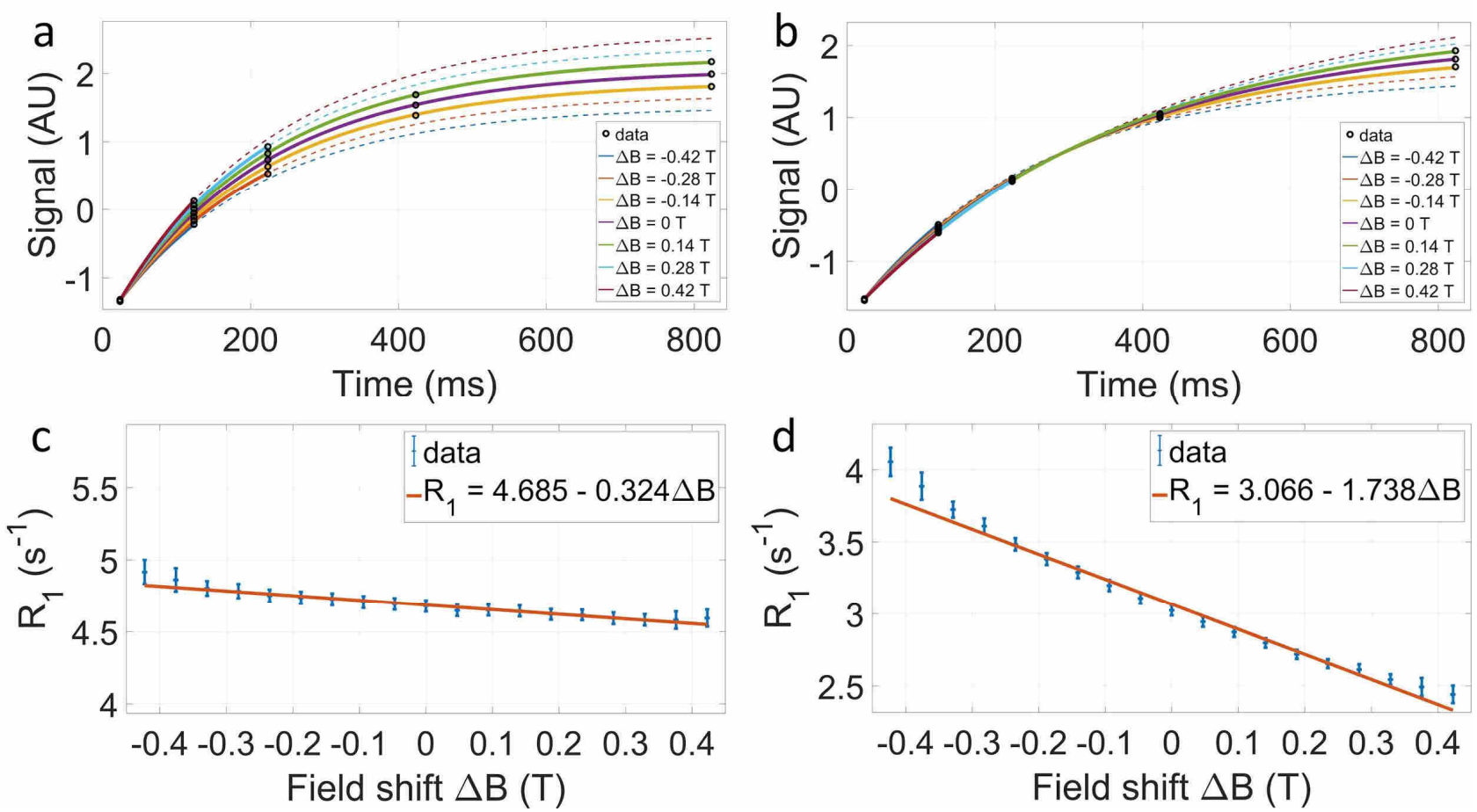

Figure 9: Exemplary measurements of $R_{1}$ NMRD profiles. FFC inversion-recovery signals plotted for a non-dispersive $1 \mathrm{mM} \mathrm{Gd-DOTA} \mathrm{sample} \mathrm{(a)} \mathrm{and} \mathrm{a} \mathrm{dispersive} 200 \mu \mathrm{M}$ USPIO sample (b). The measured signals for seven field offsets and several inversion time (black circles) together with the fitted recovery curves (solid line plotted between the measured points, dashed lines representing the extrapolated recovery). The non-dispersive sample (a) displays recovery curves with similar time constants tending towards a different thermal equilibrium. The dispersive sample (b) shows a slower recovery at a higher field. This is confirmed quantitatively (respectively in $c$ and $d$ for the nondispersive and dispersive samples), the linear fit (red solid line, equation given in the figure, units are $s^{-1}$ ) to the fitted relaxation rates (blue error bars indicating the $95 \%$ confidence interval) indicating a small dispersion for Gd-DOTA and a larger one for USPIO. 
Table 1: At $1.5 T$, relaxation rate $R_{10}$ and slope of the dispersion profile $\beta_{1}$ obtained by fitting the NMRD profiles in the range -10 to $10 \mathrm{MHz}$ when it is well approximated by a first order polynomial. The number in the parenthesis corresponds to the Cramer-Rao lower bound (square root of the diagonal element of the noise covariance matrix) obtained by estimating the variance of the measurement from the residual between the data and the model. The number after the \pm symbol is the standard deviation over the number of measurements (N). Data are reported for water, three samples of Gd-DOTA and USPIO (293 K).

\begin{tabular}{|c|c|c|c|}
\hline Sample & $\mathrm{R}_{10}\left(\mathrm{~s}^{-1}\right)$ & $\beta_{1}\left(\mathrm{~s}^{-1} \mathrm{~T}^{-1}\right)$ & $\mathrm{N}$ \\
\hline Water & $0.357 \pm 0.017( \pm 0.004)$ & $-0.039 \pm 0.002( \pm 0.032)$ & 5 \\
\hline Gd-DOTA $166 \mu \mathrm{M}$ & $1.04 \pm 0.003( \pm 0.002)$ & $-0.070 \pm 0.010( \pm 0.017)$ & 3 \\
\hline Gd-DOTA $333 \mu \mathrm{M}$ & $1.785 \pm 0.005( \pm 0.004)$ & $-0.127 \pm 0.015( \pm 0.029)$ & 3 \\
\hline Gd-DOTA $1 \mathrm{mM}$ & $4.66 \pm 0.07( \pm 0.07)$ & $-0.32 \pm 0.05( \pm 0.05)$ & 3 \\
\hline USPIO $60 \mu \mathrm{M}$ & $1.041 \pm 0.019( \pm 0.006)$ & $-0.463 \pm 0.005( \pm 0.039)$ & 3 \\
\hline USPIO $120 \mu \mathrm{M}$ & $1.889 \pm 0.005( \pm 0.005)$ & $-1.019 \pm 0.007( \pm 0.032)$ & 3 \\
\hline USPIO $180 \mu \mathrm{M}$ & $2.871 \pm 0.008( \pm 0.006)$ & $-1.640 \pm 0.011( \pm 0.038)$ & 3 \\
\hline
\end{tabular}

The FFC spin-echo experiment (Fig. 10) provided a stable spin-echo magnitude for the Gd-DOTA sample transverse magnetization relaxing under different field offset areas indicating a negligible transverse relaxation dispersion $\left(\mathrm{R}_{2,0}=56.76 \pm 0.03\right.$ and $\left.\beta_{2}=0.62 \pm 0.65 \mathrm{~T} . \mathrm{s}^{-1}\right)$. By contrast, spin-echo magnitude depended strongly on field offset areas for ferritin, indicating a strong transverse relaxation dispersion $\left(\mathrm{R}_{2,0}=50.71 \pm 0.01, \beta_{2}=27.13 \pm 0.49 \mathrm{~T} . \mathrm{s}^{-1}\right)$ as expected.

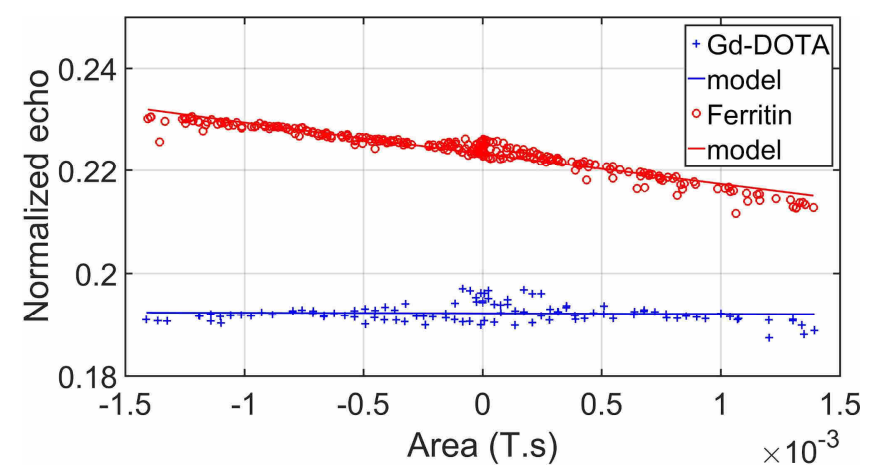

Figure 10: $R_{2}$ NMRD quantification. Normalized first echo magnitude as a function of the applied field area for the Gd-DOTA (blue crosses) and ferritin (red circles) solutions displaying a strong decrease for the ferritin sample, indicating an increase of the transverse relaxation rate when the magnetic field increases. The echo magnitudes vary almost linearly with the total area of the field offset pulses A. The fitted parameters are indicated in the text. 


\section{$\underline{R_{1} \text { dispersion mapping }}$}

Quantitative images obtained on the tubes were free of artifacts (Fig. 11), indicating that the eddycurrent compensation technique led to a frequency precision better than the RF pulse and pixel bandwidths and ensured correct slice positioning and in plane location. The tubes presented homogeneous $\mathrm{R}_{1,0}$ and $\beta_{1}$, with values that were the same (within uncertainties) as the ones obtained with the spectroscopic mode (Tables 1 and 2) when available (for water and both USPIO samples). For the tubes, $\mathrm{R}_{10}$ between 0.3 and $1.3 \mathrm{~s}^{-1}$ could be measured with a precision on the order of $0.05 \mathrm{~s}^{-1}$, and $\beta_{1}$ between -0.6 and $0 \mathrm{~s}^{-1} \mathrm{~T}^{-1}$ with a precision on the order of $0.07 \mathrm{~s}^{-1} \mathrm{~T}^{-1}$.

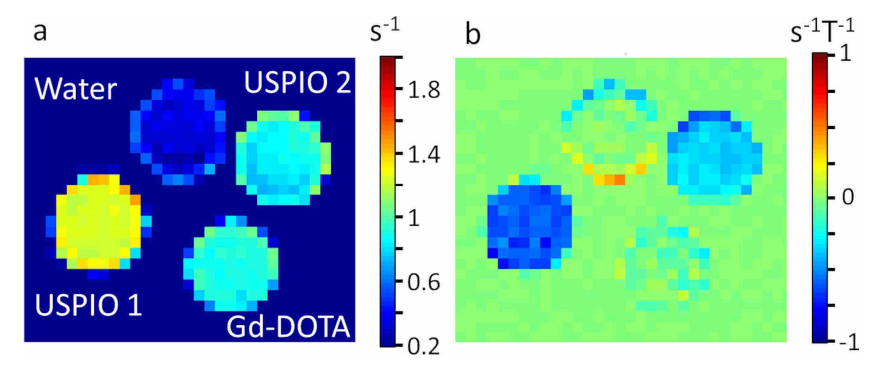

Figure 11: Imaging results. Relaxation rates at $1.5 T R_{1,0}(a)$ and $\beta_{1}$ map $(b)$ for various solutions (Water, USPIO and Gd-DOTA). The tubes corresponded to low-dispersive water and Gd-DOTA samples, and two dispersive USPIO samples (negative value of $\beta_{1}$ ).

Table 2: Relaxation rate and dispersion quantification from images. Mean and standard deviation over the ROIs of relaxation rate at $1.5 T R_{10}$ and slope of the dispersion profile. The number $N$ is the number of points inside each ROI used for the calculation. The values in parenthesis provided for the USPIO samples are the results of the spectroscopic measurements for which the indicated uncertainty is the Cramer-Rao lower bound.

\begin{tabular}{|c|c|c|c|}
\hline ROI & $\mathrm{R}_{10}\left(\mathrm{~s}^{-1}\right)$ & $\beta_{1}\left(\mathrm{~s}^{-1} \mathrm{~T}^{-1}\right)$ & $\mathrm{N}$ \\
\hline Water & $0.359 \pm 0.066$ & $-0.022 \pm 0.085$ & 50 \\
\hline Gd-DOTA & $0.920 \pm 0.053$ & $-0.054 \pm 0.078$ & 52 \\
\hline USPIO 1 & $\begin{array}{c}1.252 \pm 0.036 \\
(1.194 \pm 0.034)\end{array}$ & $\begin{array}{c}-0.594 \pm 0.047 \\
(-0.585 \pm 0.046)\end{array}$ & 49 \\
\hline USPIO 2 & $\begin{array}{c}0.853 \pm 0.065 \\
(0.825 \pm 0.034)\end{array}$ & $\begin{array}{c}-0.344 \pm 0.061 \\
(-0.321 \pm 0.005)\end{array}$ & 49 \\
\hline
\end{tabular}




\section{DISCUSSION}

In this work, a device for FFC relaxometry and imaging around $1.5 \mathrm{~T}$ was presented, together with characterization and validation methods. Precision and stability, as well as homogeneity and eddycurrent compensation were reported. $\mathrm{R}_{1}$ and $\mathrm{R}_{2} \mathrm{NMRD}$ profiles could be quantified from 1.08 to 1.92 $T$, essentially displaying a linear evolution in this range. $\mathrm{R}_{1}$ NMRD profiles were measured on various samples and contrast agent solutions, providing results consistent with literature. $\mathrm{R}_{2}$ dispersion could be measured at high magnetic field using a FFC insert technology, and a large dispersion was measured on a ferritin solution, consistently with previously reported data acquired at different static fields. Quantitative dispersive images $\left(\beta_{1}\right)$ could be generated on solutions, with specific hardware, eddy-current compensation, imaging protocol and reconstruction methods enabling to achieve good image quality and quantitative results consistent with the spectroscopic FFC measurements. As the system is large enough for small animal such as mice, these results open the way to quantitative preclinical dispersion imaging studies around high magnetic field.

\section{System control and performance}

The system can be described to a good approximation by a inductance-resistance circuit model with $2.625 \mathrm{~ms}$ response time when connected directly to the amplifier without the diode pair. It was chosen to drive it in voltage mode without specific feedback on the current. This limited the possibility of hazard, as the maximum voltage to reach $18 \mathrm{MHz}$ in 2-ms ramp time was on the order of $40 \mathrm{~V}$. This led to controlling directly the required voltage needed to obtain a given current and field offset, which was possible and precise using controlled over-voltages, as exemplified with trapezoidal waveforms. Without the diode pair, random current fluctuations were observed, having a significant effect on the signal frequency and phase, impeding the raw accumulation of FIDs from multiple acquisitions, and in particular leading to phase artefacts during the various k-space lines acquisition needed for imaging. For imaging, the phase fluctuation can be corrected using specific estimation and reconstruction approaches ${ }^{30}$. Here, as the detection is performed using a stable superconductive magnet, two hardware solutions were used. The first one is based on disabling the amplifier ${ }^{32}$, as was done here for longitudinal relaxation dispersion measurements. Indeed, the repetition times required in such sequence were long ( $>1 \mathrm{~s}$ ) and compatible with the time needed to enable the amplifier again (350 ms). The second solution is the use of the diode pair: then the amplifier could be kept enabled for long periods, and large waveforms could be repeated in a short repetition time interleaving them with RF pulses and acquisition periods as in the transverse relaxation dispersion measurements. The diode pairs reduced the current fluctuation in a range compatible with RF excitation and signal detection, while keeping the ability to reach large field offsets. 
Adding the diode pair had the effect of modifying the equivalent resistance of the system, rendering it non-linear with the voltage: it was increased by 56 times for low currents, and only by $4 \%$ for large currents. This reduces the characteristic time for low currents (typically lower than $62.5 \mathrm{~mA}$ ), leading to a faster return to zero for the detection.

Applying $0.2 \mathrm{~T}(8.5 \mathrm{MHz})$ continuously $(\sim 2.1 \mathrm{~kW}$ dissipated as heat) resulted in a $10 \mathrm{~K}$ increase of the overheating sensor, chosen as the safety limit to shut-down the system. In terms of applicable field shift and duration, the applied waveforms were thus limited here to $1.5 \mathrm{~kJ}$ dissipated as heat into the insert chosen to avoid untimely system security shut-down during repeated experiments. For the maximum field offset reported here (18 MHz NMR frequency, $9.6 \mathrm{~kW}$ power), this limited the application time to a maximum of $160 \mathrm{~ms}$, still enabling sensitizing to dispersive properties at this field shift. To apply higher and longer waveforms while limiting the insert absolute temperature below critical values, a specific water chiller could be used instead of the lost-water setup that was used here. .

Regarding the accuracy in the reported field shift value, the experiments reported in Fig. 5 and 7 enabled us to estimate it to be better than $\pm 0.01 \mathrm{~T}$ for all the applied waveform $(2 \%$ of the range of $\pm 0.48 \mathrm{~T}$ ), even considering the resistance increase as a result of the small dynamic temperature change when a waveform was applied. Driving the system with an adequate current feedback would be a technical solution to improve the accuracy of the waveforms regardless of the resistance changes. However, in our preliminary experience, this led to a reduced precision of the current during detection with effects that became larger than the ones displayed in Fig. 6 a and c. This is indeed difficult to have a feedback system regulating accurately (on the order of 1\%) the current during non-zero waveforms, while keeping precise (on the order of $1 \mathrm{ppm}$ ) values during detection.

\section{Frequency measurement, eddy-current and compensation for imaging}

A method for the measurement of frequency based on phase derivation was proposed. It allowed measuring NMR frequency variations in the range $\pm 500 \mathrm{kHz}$ corresponding to the maximum acquisition bandwidth of the pulse sequencer. It provided instantaneous measurement with a precision that depended on the chosen effective filter bandwidth. Indeed, as NMR frequencies are not expected to vary at frequencies higher than the ones filtered by the system $(60 \mathrm{~Hz}$ with $0.1 \Omega, 3.4 \mathrm{kHz}$ with $6.4 \Omega$ ), a 2-to-10 kHz bandwidth could be applied, resulting in precision on the order of 1 to $10 \mathrm{~Hz}$ when SNR from a single FID was sufficient (SNR 30 with a 1-MHz acquisition bandwidth). However, this limited the continuous measurement of frequency for a time corresponding to the FID 
signal duration. This led to repeat the experiment sequentially increasing the delay between the field pulse and the FIDs, or to repeat rapidly FID acquisitions with a small RF pulse angle for faster acquisition. The former method ensured a pseudo-continuous reconstitution of frequency as a function of time after a field offset pulse, while the latter could suffer from FID signal cancelling due to destructive effects of stimulated echoes and had unmeasured period of time (to apply RF pulses) between FID acquisitions. To avoid such episodic effects and enable the recovery of the full temporal evolution, an adaptive filter (e.g Kalman filter) accounting for the current frequency measurement and the previous estimation could be implemented.

Both approaches provided consistent measure of the eddy current, with effects that were linear with the field offset pulse amplitude. Amplitude were on the order of $150 \mathrm{~Hz} / \mathrm{MHz} 12 \mathrm{~ms}$ after the end of a $500 \mathrm{~ms}$ pulse, and with a time constant on the order of $100 \mathrm{~ms}$. In each case, the eddy-current decay could be modelled with a dual exponential decay, providing time constant and amplitude that modelled the frequency decay better but that were less consistent between the pseudo-continuous and the fast methods. This may be due to the slight differences between the two experiments reported here (step voltage command versus trapezoidal waveform, and acquisition starting $12 \mathrm{~ms}$ versus $5 \mathrm{~ms}$ after the waveform) or more probably to the limited stability on the parameters estimates when fitting to a dual exponential model. It was chosen here to apply directly a counter-waveform using the low power auxiliary amplifier in order to reduce the eddy-currents, not relying on a model but directly on the measured frequency. Compensation enabled to reach field stability, with values below $50 \mathrm{~Hz}$ for a period covering $12 \mathrm{~ms}$ to $500 \mathrm{~ms}$ corresponding to the window where images were formed. Indeed, given the chosen imaging parameters, $50 \mathrm{~Hz}$ is small as compared to usual RF pulses bandwidth (in the $\mathrm{kHz}$ range) and to the pixel bandwidth that was used for the FFC-IR imaging experiment (200 $\mathrm{Hz}$ ) ensuring limited slice mispositioning and in-plane shifts. The typical range for correcting the eddy-current (below $2 \mathrm{kHz}$ ) required low current $(35 \mathrm{~mA}$ ) that could be achieved easily with a small amplifier using $100 \Omega$ and $3.5 \mathrm{~V}$. One practical limitation of the auxiliary amplifier implemented is that it was monopolar, which required inverting the connection for positive and negative corrections (respectively for positive and negative field offsets). Bipolar systems could be implemented easily to avoid this manual intervention.

Eddy currents could also be reduced using a shielding strategy, as was followed by other groups $^{20,24,28,32}$. However, this is done at the expense of the efficiency, and it is hard though to compare the various designs as the insert bore sizes and host MRI systems differ ${ }^{33}$. Given the reported literature data and the present ones, the eddy-current amplitude ranged between 15 to $150 \mathrm{~Hz} / \mathrm{MHz}$ 
with time constants on the order of a hundred to few hundreds of milliseconds.

\section{Homogeneity}

The spatial homogeneity of the system was better than 2500 ppm peak-peak, 270 ppm RMS inside a 25-mm diameter sphere targeted for sample/animal size. This is a very small relative dispersion $(0.25 \%)$ leading to homogenous and precise field offset values. This is sufficient for relaxometric NMRD profile measurements at high field here, as, even for the largest field offsets of $\pm 18 \mathrm{MHz}$ NMR frequency probed here, centered on $63.8 \mathrm{MHz}$, spatial variations up to $45 \mathrm{kHz}$ peak-peak, 5 $\mathrm{kHz}$ RMS are expected, a range on which $\mathrm{R}_{1}$ and $\mathrm{R}_{2}$ can be considered not to vary significantly. However, this leads to spatial phase dispersion such that spin-echoes were required for FFC transverse relaxation measurements to cancel the accumulated phase shifts. The gradient over this region can roughly be estimated to be $1077 \mathrm{ppm}$ over $9 \mathrm{~mm}$ (as the 18-mm diameter sphere was used). For the maximum field offset of $0.353 \mathrm{~T}$ applied for the transverse relaxation measurements, it corresponds to $42 \mathrm{mT} . \mathrm{m}^{-1}$ approximately. This gradient can also induce additional diffusion attenuation ${ }^{45}$, that depends on its square (and thus on the square of the applied field area in Fig. 10), its application time and delay between the pulses, with effects that are spatially dependent as the gradient is not uniform. Considering the FFC spin-echo sequence timings, diffusion weighting in such a field gradient for free water would lead to a moderate attenuation of the spin echo by $1.7 \%$, negligible as compared to the attenuation due to transverse relaxation. Additionally, the exact balancing of field offset sections (i.e. before and after the refocusing RF pulses) during FFC spin-echo experiments are critical to produce an echo. Indeed, due to the field inhomogeneities produced by the insert, unbalanced areas lead to an incomplete refocusing at the echo time. By enforcing that only the acquisitions with a difference of less than $2 \times 10^{-6}$ T.s were considered, with a inhomogeneity dispersion of $147 \mathrm{ppm}$, this ensured that the phase dispersion at the echo time was less than $0.08 \mathrm{rad}$ with negligible effects on the echo amplitude (estimated to result in a negligible signal attenuation of less than 1\%).

\section{$\underline{R}_{1}$ and $R_{2}$ NMRD measurements}

Dispersion measurements were done using standard inversion-recovery (for $\mathrm{R}_{1}$ ) and spin-echo (for $\mathrm{R}_{2}$ ) sequences, with relaxation periods spent partially at different fields. For the contrast agents and samples studied here, the NMRD profiles (directly for $\mathrm{R}_{1}$, and indirectly for $\mathrm{R}_{2}$ as it can be extracted from echo attenuation) appeared linearly varying with $\mathrm{B}_{0}$. The slopes $\beta_{1}$ and $\beta_{2}$ corresponding to the first order derivative of, respectively, the longitudinal and transverse relaxation rates with respect to the magnetic field at $1.5 \mathrm{~T}$, and the NMRD profiles could be summarized by a first order polynomial around 1.5 T. This linear behaviour is expected for these types of contrast agents and samples ${ }^{13-15,46}$, but that may not be the case in specific situations such as when using specific contrasts agents 
exploiting quadrupole relaxation enhancement ${ }^{47}$. The obtained values both for $\mathrm{R}_{1}$ and $\mathrm{R}_{2}$ dispersion are consistent with previously published data (see appendix)

The results presented here validate the presented hardware and methods to probe longitudinal NMRD profiles between 1.08 to $1.92 \mathrm{~T}$, as well as the measurement of the parameters of a simplified linear approximation around $1.5 \mathrm{~T}$ both for longitudinal and transverse relaxation rates. Longitudinal relaxation rates typically in a range $0.3-3 \mathrm{~s}^{-1}$ could be measured with a precision better than $0.01 \mathrm{~s}^{-1}$, and dispersion slope typically in a range of -2 to $0 \mathrm{~s}^{-1} \mathrm{~T}^{-1}$ with a precision better than $0.05 \mathrm{~s}^{-1} \mathrm{~T}^{-1}$. The only exception was for the $1 \mathrm{mM}$ Gd-DOTA for which less precise measurements were obtained as a consequence of its larger relaxation rates and to the sampled inversion times applied here. Transverse dispersions in the range 0 to $30 \mathrm{~s}^{-1} \mathrm{~T}^{-1}$ could be measured with a precision better than $1 \mathrm{~s}^{-}$ ${ }^{1} \mathrm{~T}^{-1}$ for solutions presenting a large relaxation rate at $1.5 \mathrm{~T}$, close to $50 \mathrm{~s}^{-1}$. Only the first spin-echo signal was analysed here, but the second echo also presented measurable dispersive effects suggesting the use of spin-echo trains to accumulate field shifts over a longer period and to generate larger transverse dispersion effects. This would allow increasing precision and to access to smaller relaxation rates. The ability of our system to balance field shift areas over a longer period and obtain reproducible echo trains for more than $60 \mathrm{~ms}$, however, still needs to be tested. Nevertheless, these results present an important step towards the precise measurements for samples with transverse relaxation rates closer to the one encountered for tissues in vivo $\left(\sim 1-10 \mathrm{~s}^{-1}\right)$ and towards a transfer to transverse relaxation dispersion mapping.

\section{$\underline{R_{1} N M R D \text { mapping performances }}$}

The dispersive images on the tested solutions provided the same quantitative results as the spectroscopic measurements, validating the imaging protocol as well as the developed data processing for fitting the data to the Bloch equations. Inversion time was chosen in a range adapted to the expected relaxation rates, so as to obtain a good precision. The range and precisions obtained for longitudinal relaxation rate as well as for the slope of the dispersion profile were essentially similar to the values reported for the spectroscopic experiments, but with much less sample volumes, as voxel size were on the order of $0.63 \mu \mathrm{L}$, demonstrating that the combined setup, protocol and data analysis enable localized measurements of spatially heterogeneous samples for a 2D slice in a protocol lasting in total less than 25 minutes providing a typical precision of $0.05-0.1 \mathrm{~s}^{-1} \mathrm{~T}^{-1}$. For comparison with previous FFC-MRI relaxometric measurements with FFC-MRI inserts at $1.5 \mathrm{~T}$ and $3 \mathrm{~T}$, Araya et al. ${ }^{31}$ acquired $2 \mathrm{D}$ slices with voxel volumes of $0.44 \mu \mathrm{L}$ using a fast spin echo imaging sequence at various inversion times and magnetic fields in a range of $\pm 0.24 \mathrm{~T}$ around $1.5 \mathrm{~T}$ leading to approximately 2 
hours scan time, and Bödenler et al. ${ }^{32,48}$ acquired 2D slices with voxel volumes of $1.95 \mu \mathrm{L}$ with a single spin echo sequence with several inversion times and magnetic fields in the range $\pm 0.1 \mathrm{~T}$ around $3 \mathrm{~T}$ in 22.4 to $85 \mathrm{~min}$, reporting typical precisions (differences between the 3rd and 1st quartiles over samples $^{48}$ ) of $0.15 \mathrm{~s}^{-1} \mathrm{~T}^{-1}$. Generally speaking, the precision of longitudinal relaxation dispersion mapping can be enhanced by using smaller RF coils or sequences exploiting more efficiently the dead times, e.g. using fast spin echo ${ }^{29}$. Optimizing the probed inversion times as well as increasing the field offsets for targeted range of relaxation rates is also a possibility to reduce scan time down to scan durations more adapted to in vivo imaging.

\section{CONCLUSION}

To conclude, we presented the integration and characterization of a FFC insert into a $1.5 \mathrm{~T}$ MRI system with specific calibration and compensation approaches. System homogeneity, precision and stability were assessed using specifically designed measurements based on NMR frequency. A diode pair was proposed to reduce the current fluctuation during detection while maintaining large field offset capabilities during relaxation. A dual-amplifier strategy was used to compensate eddy-current fluctuations. Longitudinal NMRD profile measurements was shown possible in a limited range around $1.5 \mathrm{~T}$, and a linear approximation of the relaxation profiles in which the dispersive information is reduced to the slope of the relaxation profile was validated. An imaging protocol and associated reconstruction methods permitted to obtain longitudinal relaxation dispersion mapping in conditions compatible with in vivo experiments. The range for transverse relaxation dispersion measurements was also extended to fields higher than previously reported, a step towards transverse relaxation dispersion mapping at high-field. The capabilities in terms of range and precision of such new parameters suggest that relaxation dispersion around 1.5 $\mathrm{T}$ could be quantified and imaged in vivo in small animal with a precision sufficient to detect small endogenous differences between tissues.

ACKNOWLEDGMENTS: This work was supported by the COST Action CA15209 European Network on NMR Relaxometry (EURELAX). This work was partly funded by the French program "Investissement d'Avenir" run by the 'Agence Nationale de la Recherche'; the grant reference is 'Infrastructure d'avenir en Biologie Santé - ANR-11-INBS-0006'. FFC-MRI experiments were done on the 1.5 T MRI platform of SHFJ, Frédéric Joliot Institute for Life Sciences (ANR-11-INBS-0006). The authors would like to thank G. Ferrante, M. Polello, R. Rolfi from Stelar s.r.l for technical support, as well as B. Rutt and E. Lee (Radiological Sciences Lab., Stanford Univ.) for initial tests of the FFC insert (2011 France-Stanford collaborative project), and Dr. Lionel Broche (Aberdeen Biomedical Imaging Centre, University of Aberdeen) for helpful discussions during his visit as an invited researcher at Paris-Sud Univ. in June 2017. 


\section{REFERENCES}

${ }^{1}$ F. Bloch, Phys. Rev. 70, 460 (1946).

${ }^{2}$ M.H. Levitt, Spin Dynamics (John Wiley \& Sons, Chichester, New York, 2001).

${ }^{3}$ A.G. Redfield, IBM J. Res. Dev. 1, 19 (1957).

${ }^{4}$ A. Abragam, The Principles of Nuclear Magnetism (Oxford university Press, New York, 1961).

${ }^{5}$ M. Goldman, J. Magn. Reson. 149, 160 (2001).

${ }^{6}$ R. Kimmich and E. Anoardo, Prog. Nucl. Magn. Reson. Spectrosc. 44, 257 (2004).

${ }^{7}$ R. Kimmich, editor , Field-Cycling NMR Relaxometry: Instrumentation, Model Theories and Applications (The Royal Society of Chemistry, 2019).

${ }^{8}$ O. Lips, A.F. Privalov, S.V. Dvinskikh, and F. Fujara, J Magn Reson 149, 22 (2001).

${ }^{9}$ Y. Gossuin, Z. Serhan, L. Sandiford, D. Henrard, T. Marquardsen, R.T.M. de Rosales, D. Sakellariou, and F. Ferrage, Appl. Magn. Reson. 47, 237 (2016).

${ }^{10}$ R.M. Steele, J.-P. Korb, G. Ferrante, and S. Bubici, Magn. Reson. Chem. MRC 54, 502 (2016).

${ }^{11}$ S. Kruber, G.D. Farrher, and E. Anoardo, J. Magn. Reson. San Diego Calif 1997 259, 216 (2015).

${ }^{12}$ S.H. Koenig and R.D. Brown, Magn. Reson. Med. 1, 437 (1984).

${ }^{13}$ G. Diakova, J.-P. Korb, and R.G. Bryant, Magn. Reson. Med. 68, 272 (2012).

${ }^{14}$ Y. Gossuin, A. Roch, R.N. Muller, and P. Gillis, Magn. Reson. Med. 43, 237 (2000).

${ }^{15}$ Q.L. Vuong, P. Gillis, A. Roch, and Y. Gossuin, Wiley Interdiscip. Rev. Nanomed. Nanobiotechnol. 9, (2017).

${ }^{16}$ P.A. Rinck, H.W. Fischer, L. Vander Elst, Y. Van Haverbeke, and R.N. Muller, Radiology 168, 843 (1988).

${ }^{17}$ L.M. Broche, S.R. Ismail, N.A. Booth, and D.J. Lurie, Magn Reson Med 67, 1453 (2012).

${ }^{18}$ L.M. Broche, G.P. Ashcroft, and D.J. Lurie, Magn Reson Med 68, 358 (2012).

${ }^{19}$ A.M. Oros-Peusquens, M. Laurila, and N.J. Shah, MAGMA 21, 131 (2008).

${ }^{20}$ J.K. Alford, B.K. Rutt, T.J. Scholl, W.B. Handler, and B.A. Chronik, Magn. Reson. Med. 61, 796 (2009).

${ }^{21}$ D.J. Lurie, M.A. Foster, D. Yeung, and J.M. Hutchison, Phys Med Biol 43, 1877 (1998).

${ }^{2}$ S.E. Ungersma, N.I. Matter, J.W. Hardy, R.D. Venook, A. Macovski, S.M. Conolly, and G.C. Scott, Magn. Reson. Med. 55, 1362 (2006).

${ }^{23}$ K.J. Pine, G.R. Davies, and D.J. Lurie, Magn Reson Med 63, 1698 (2010).

${ }^{24}$ U.C. Hoelscher, S. Lother, F. Fidler, M. Blaimer, and P. Jakob, MAGMA 25, 223 (2012).

${ }^{25}$ U.C. Hoelscher and P.M. Jakob, MAGMA 26, 249 (2013).

${ }^{26}$ K.J. Pine, F. Goldie, and D.J. Lurie, Magn Reson Med 72, 1492 (2014).

${ }^{27}$ L.M. Broche, P.J. Ross, K.J. Pine, and D.J. Lurie, J. Magn. Reson. 238, 44 (2014).

${ }^{28}$ C.T. Harris, W.B. Handler, Y. Araya, F. Martínez-Santiesteban, J.K. Alford, B. Dalrymple, F. Van Sas, B.A. Chronik, and T.J. Scholl, Magn. Reson. Med. 72, 1182 (2014).

${ }^{29}$ P.J. Ross, L.M. Broche, and D.J. Lurie, Magn. Reson. Med. 73, 1120 (2015).

${ }^{30}$ L.M. Broche, P.J. Ross, G.R. Davies, and D.J. Lurie, Magn. Reson. Imaging 44, 55 (2017).

31 Y.T. Araya, F. Martínez-Santiesteban, W.B. Handler, C.T. Harris, B.A. Chronik, and T.J. Scholl, NMR Biomed. 30, e3789 (2017).

${ }^{32}$ M. Bodenler, M. Basini, M.F. Casula, E. Umut, C. Gosweiner, A. Petrovic, D. Kruk, and H. Scharfetter, J Magn Reson 290, 68 (2018).

${ }^{33}$ M. Bödenler, L. de Rochefort, P.J. Ross, N. Chanet, G. Guillot, G.R. Davies, C. Gösweiner, H. Scharfetter, D.J. Lurie, and L.M. Broche, Mol. Phys. 1 (2018).

${ }^{34}$ N. Chanet, G. Guillot, I. Leguerney, R.-M. Dubuisson, C. Sebrié, A. Ingels, N. Assoun, E. Daudigeos-Dubus, B. Geoerger, N. Lassau, L. Broche, and L. de Rochefort, Proc Intl Soc Mag Reson Med 2262 (2018).

${ }^{35}$ R. Kimmich, editor, in Field-Cycl. NMR Relaxometry Instrum. Model Theor. Appl. (The Royal Society of Chemistry, 2019), pp. 358-384.

${ }^{36}$ L. de Rochefort, E. Lee, M. Pollelo, L. Darrasse, G. Ferrante, and B. Ruth, Proc Intl Soc Mag Reson Med 4165 (2012).

${ }^{37}$ N. De Zanche, C. Barmet, J.A. Nordmeyer-Massner, and K.P. Pruessmann, Magn. Reson. Med. 60, 176 (2008).

${ }^{38}$ D.J. Lurie, S. Aime, S. Baroni, N.A. Booth, L.M. Broche, C.-H. Choi, G.R. Davies, S. Ismail, D.O. Hogain, and K.J. Pine, Comptes Rendus Phys. 11, (2010).

${ }^{39}$ J. Mispelter, M. Lupu, and A. Briguet, NMR Probeheads for Biophysical and Biomedical Experiments, Imperial College Press (2006).

40 T.E. Conturo and G.D. Smith, Magn. Reson. Med. 15, 420 (1990).

${ }^{41}$ L. de Rochefort, R. Brown, M.R. Prince, and Y. Wang, Magn. Reson. Med. 60, 1003 (2008).

${ }^{42}$ M. Rohrer, H. Bauer, J. Mintorovitch, M. Requardt, and H.-J. Weinmann, Invest. Radiol. 40, 715 (2005).

${ }^{43}$ E. Goldammer and W. Kreysch, Berichte Bunsen-Ges.-Phys. Chem. Chem. Phys. 82, 463 (1978).

${ }^{44}$ F. Noack, Prog. Nucl. Magn. Reson. Spectrosc. 18, 171 (1986). 
${ }^{45}$ G. Ferrante, D. Canina, E. Bonardi, M. Polello, S. Sykora, P. Golzi, C. Vacchi, and R. Stevens, 5th Conf. Field Cycl. NMR Relaxometry Torino Italy (2007).

${ }^{46}$ S. Laurent, L.Vander Elst, and R.N. Muller, Contrast Media Mol. Imaging 1, 128 (2006).

${ }^{47}$ D. Kruk, E. Masiewicz, E. Umut, A. Petrovic, R. Kargl, and H. Scharfetter, J. Chem. Phys. 150, 184306 (2019).

${ }^{48}$ M. Bödenler, K.P. Malikidogo, J.-F. Morfin, C.S. Aigner, É. Tóth, C.S. Bonnet, and H. Scharfetter, Chemistry 25, 8236 (2019). 


\section{APPENDIX 1 - Derivation of the transverse relaxation rate decay}

In this section, we express the transverse decay induced by a field cycling pulse during the spin echo as depicted in Fig. 4. We start from the evolution of the transverse magnetization right after a $90^{\circ}$ pulse, assuming a variation of the magnetic field as a function of time $t$ :

$$
\frac{d M}{d t}(t)=-R_{2}(B(t)) \times M(t)
$$

This equation has the following solution:

$$
M(T E)=M(0) \times \exp \left(-\int_{0}^{T E} \quad R_{2}(B(t)) d t\right),
$$

leading to the signal at the echo time :

$$
M(T E)=M(0) \times \exp \left(-R_{2,0} T E\right) \exp \left(-\beta_{2} A\right)
$$

if we assume a first order approximation:

$$
R_{2}=R_{2,0}+\beta_{2}\left(B(t)-B_{0}\right)
$$

with:

$$
A=\int_{0}^{T E}\left(B(t)-B_{0}\right) d t
$$

If the variations are small, i.e. $\beta_{2} A<<1$, then the signal variation is linear with $A$ providing a simpler linear relationship:

$$
M(T E)=M(0) \times \exp \left(-R_{2,0} T E\right) \times\left(1-\beta_{2} A\right) .
$$




\section{APPENDIX 2 - Dispersion comparison with literature data}

\section{$\underline{R_{1}}$ dispersion consistency with literature}

For tap water, a small $\mathrm{R}_{10}$ was measured, slightly higher than the expected high-field limit for bulk water ${ }^{13}$, together with a small dispersion indicating a trend to decrease with magnetic field.

For Gd-DOTA, the longitudinal molar relaxivity at $1.5 \mathrm{~T}$ in water at $293 \mathrm{~K}\left(4.31 \mathrm{mM}^{-1} \mathrm{~s}^{-1}\right)$ as well as its slope $\left(-0.282 \mathrm{mM}^{-1} \mathrm{~s}^{-1} \mathrm{~T}^{-1}\right)$ are consistent with reported data ${ }^{41,46}$. From these literature data, we can roughly extrapolate a variation with magnetic field of $-0.22 \mathrm{mM}^{-1} \mathrm{~s}^{-1} \mathrm{~T}^{-1}$ at $310 \mathrm{~K}$ (by fitting the reported data to a line), with a slope expected to be slightly more pronounced at $293 \mathrm{~K}$ in the present work. Indeed, Laurent et al. ${ }^{46}$ reported molar relaxivities of 3.5 and $3.1 \mathrm{mM}^{-1} \mathrm{~s}^{-1}$ in water at $310 \mathrm{~K}$ at 0.47 and $1.41 \mathrm{~T}$, respectively, as well as a value close to $4.8 \mathrm{mM}^{-1} \mathrm{~s}^{-1}$ at $0.47 \mathrm{~T}$ and $293 \mathrm{~K}$. Rohrer et $\mathrm{al}^{41}$ reported values for Gd-DOTA in water at $310 \mathrm{~K}$ of 3.4 (3.2-3.6), 2.9 (2.7-3.1) and 2.8 (2.6-3.0) $\mathrm{mM}^{-1} \mathrm{~s}^{-1} \mathrm{~T}^{-1}$ at $0.47,1.5$ and $3 \mathrm{~T}$ respectively.

Regarding the $30 \mathrm{~nm}$ USPIO particle, the longitudinal molar relaxivity at $1.5 \mathrm{~T}$ in water at $293 \mathrm{~K}$ (14.4 $\left.\mathrm{mM}^{-1} \mathrm{~s}^{-1}\right)$ as well as its slope $\left(-9.14 \mathrm{mM}^{-1} \mathrm{~s}^{-1} \mathrm{~T}^{-1}\right)$ are in the expected range for such superparamagnetic nanoparticles, displaying a steep decrease of relaxivity around $1.5 \mathrm{~T}$. The manufacturer reported a molar relaxivity in water of $36.4 \mathrm{mM}^{-1} \mathrm{~s}^{-1}$ at $310 \mathrm{~K}$ and $0.47 \mathrm{~T}$. Bödenler et al. ${ }^{32}$ obtained values around roughly 21 and $10 \mathrm{mM}^{-1} \mathrm{~s}^{-1}$ at $1.5 \mathrm{~T}$ (by reading the available curves for S8_hex and C8_hex). At $3 \mathrm{~T}$, they obtained values of 13.17 and $6.07 \mathrm{mM}^{-1} \mathrm{~s}^{-1}$ with slopes of -5.35 and $-1 ., 6 \mathrm{mM}^{-1} \mathrm{~s}^{-1} \mathrm{~T}^{-1}$ (by fitting the provided data by a line) for respectively the two tested particles. The theory predicts a very strong decrease in a range $\sim 10 \mathrm{MHz}-200 \mathrm{MHz}$, although it depends on the particle properties (such as core size, particle size and coating) ${ }^{48}$. In any case, a steeper decrease is expected around $1.5 \mathrm{~T}$ than $3 \mathrm{~T}$ for USPIO.

\section{$\underline{R}_{2}$ dispersion consistency with literature}

The measurement of transverse relaxation dispersion was shown possible around $1.5 \mathrm{~T}$, providing negligible dispersion for Gd-DOTA and a strong one for ferritin. Other groups presented similar measurement protocol, but at different fields: between $23 \mathrm{mT}$ and $46 \mathrm{mT}^{42,43}$, and more recently up to $0.47 \mathrm{~T}^{44}$. While limited in its range in the vicinity of $1.5 \mathrm{~T}$, the presented system extends the measurement capability for transverse relaxation dispersion. Neglecting the contribution of water for the concentrations used here, the transverse relaxivity can be estimated to be $0.048 \pm 0.050 \mathrm{mM}^{-1} \mathrm{~s}^{-1} \mathrm{~T}^{-}$ ${ }^{1}$ for Gd-DOTA and $0.160 \pm 0.003 \mathrm{mM}^{-1} \mathrm{~s}^{-1} \mathrm{~T}^{-1}$ for ferritin, consistently with literature. Indeed, Rohrer 
et al. reported values in water at $310 \mathrm{~K}$ of $3.2(2.5-3.9)$ and $3.3(3.0-3.6) \mathrm{mM}^{-1} \mathrm{~s}^{-1} \mathrm{~T}^{-1}$ at 1.5 and $3 \mathrm{~T}$ respectively indicating a small variation in this range. For ferritin in solution, Gossuin et al. reported a linear variation in the range $0.23-11.7 \mathrm{~T}$ with a slope of $0.166 \mathrm{mM}^{-1} \mathrm{~s}^{-1} \mathrm{~T}^{-1}$ at $313 \mathrm{~K}^{14}$. 